\title{
Cellular and Circuit Organization of the Locus Coeruleus of Adult Mice
}

Andrew McKinney 1,2,3, Ming Hü2,3, Amber Hoskins ${ }^{5}$, Arian Mohammadyar ${ }^{5}$, Nabeeha Naeem ${ }^{5}$, Junzhan Jing $^{2,3}$, Saumil S. Patel ${ }^{2}$, Bhavin R. Sheth ${ }^{6,7 *}$, Xiaolong Jiang ${ }^{1,2,3,4 *}$

${ }^{1}$ Neuroscience Graduate Program, Baylor College of Medicine, Houston, TX, USA

2Department of Neuroscience, Baylor College of Medicine, Houston, TX, USA

3Jan and Dan Duncan Neurological Research Institute at Texas Children's Hospital, Houston, TX, USA

${ }^{4}$ Department of Ophthalmology, Baylor College of Medicine, Houston, TX, USA

${ }^{5}$ University of Houston, Houston, TX, USA

${ }^{6}$ Department of Electrical \& Computer Engineering, University of Houston, Houston, TX, USA

${ }^{7}$ Center for NeuroEngineering and Cognitive Science, University of Houston, Houston, TX, USA

${ }^{*}$ Corresponding authors

\section{Abstract}

The locus coeruleus (LC) houses the vast majority of noradrenergic neurons in the brain and regulates many fundamental functions. While efferent projections of the LC have been extensively investigated, little is known about its local circuit organization. Here, we performed large-scale multi-patch recordings of LC noradrenergic neurons to profile their morpho-electric properties while simultaneously examining their interactions. LC neurons were diverse and could be classified into two major morpho-electric types. While chemical transmission was not detected, these neurons electrically connected at a rate comparable to many brain regions. Most connections form between dendrites and were restricted to narrowly spaced pairs or small clusters of neurons of the same type. Furthermore, more than two coupled pairs were often identified from individual recording sets that follow a train-like organizational pattern. The assembly of LC neurons follows a spatial and cell type-specific wiring principle that may be imposed by a unique train-like rule. 


\section{Introduction}

The locus coeruleus (LC) is a small pontine nucleus that houses the vast majority of norepinephrine (NE)-producing neurons in the brain and sends widespread noradrenergic projections to all major divisions of the central nervous system $(\mathrm{CNS})^{1}$. By releasing NE across the CNS, the LC participates in many cognitive processes and behaviors crucial for survival, including fight and flight response, attentional control, and sleep/wake cycle, and its dysregulation has been implicated in various neuropsychiatric conditions, including depression, anxiety, autism spectrum disorder, post-traumatic stress disorder, and neurodegenerative diseases ${ }^{2-5}$. Given its small size (only $\sim 1,500$ neurons in mice and 20,000 in humans in each hemisphere $)^{1,6}$, the LC has been long considered as a homogeneous cluster of NE-producing neurons (LC/NE neurons) providing a uniform global signal. However, increasing evidence supports LC/NE neurons are heterogeneous in terms of their anatomical projections, morpho-electric profiles, and specific behavioral functions they involve ${ }^{7-9}$. Particularly, recent tracing studies coupled with behavioral paradigms challenge the long-held view of the LC as a homogeneous cluster and suggest the LC is composed of multiple specialized noradrenergic modules. Each module comprises non-overlapping LC/NE neurons with specific electrophysiological properties and projection targets, thus participating in distinct functions of the circuits within its efferent domain ${ }^{9-12}$. Interestingly, LC/NE neurons within each module are also able to switch from a discrete, patterned coding mode to a global broadcast mode to cope with the more demanding situation ${ }^{12}$. Such functional divisions of the LC and their context-dependent adaptivity suggest the LC neurons are functionally heterogeneous and may interact with each other in a complex and subtle way within the local circuit to coordinate the division of labor. To understand how each LC module is functionally specialized and how distinct LC modules interact to achieve specific functions, it is essential to dissect the local circuit organization of the LC in terms of its cellular diversity and the wiring logic.

LC/NE neurons belong to monoamine neuromodulatory systems, and their axon terminals are believed to be "asynaptic" free nerve endings thought to mediate "volume transmission". Increasing evidence also suggests monoamine neurons, including LC/NE neurons, could co-release glutamate to engage fast neurotransmission ${ }^{13-15}$. It appears that NE can be released locally in the LC to activate $\alpha 2$ 
adrenoceptor ( $\alpha 2 \mathrm{AR}$ ) on the same or other LC/NE neurons to engage a negative feedback mechanism regulating their excitability ${ }^{16-19}$, but it remains to be illustrated if these neurons can interact with each other via fast neurotransmission. In addition, it is long believed that LC/NE neurons form extensive gap junctions to synchronize their activity as a global broadcast. However, gap junctions among LC/NE neurons appear to be prevalent only in neonates and decline significantly with age ${ }^{20-22}$. It remains to be determined with the direct demonstration if electrical coupling persists within adult LC neurons and whether or not synchronization of mature LC/NE neurons is mediated by gap junction ${ }^{22-25}$.

To answer all these questions, here we adopted several strategies in studying cortical circuits to the brainstem to interrogate local circuit organization of the LC in adult mice. By applying appropriate slicing protocol to obtain high-quality brainstem slices and adapting our multi-patching system to small brainstem slices ${ }^{26,27}$, we were able to perform simultaneous whole-cell recordings from up to eight mature LC neurons. By profiling morpho-electric properties of each LC/NE neuron and examining their connectivity at an unprecedented scale and level of detail (>700 neurons), we have uncovered and defined two major morpho-electric cell types of LC/NE neurons and deciphered the major mechanism governing their interactions at the single-cell resolution. Our results suggest NE released from individual LC neurons was not sufficient to activate $\alpha 2$ adrenoceptors to induce hyperpolarization, a mechanism believed to govern the interaction between LC neurons. Instead, we found electrical coupling is the major synaptic mechanism by which individual LC neurons communicate with each other, and their assembly follows a spatial and cell type-specific wiring principle that may be imposed by a unique train-like rule.

\section{Results}

\section{Electrophysiological properties of LC/NE neurons in adult mice}

To record and characterize LC neurons from adult mice, we adopted an NMDG-based slicing protocol designed for adult animals to the brainstem ${ }^{26,27}$. This approach worked very well, and the brainstem slices prepared with this method were high-quality and contained sufficient healthy LC neurons in each slice, allowing for simultaneous patch-clamp recordings of up to eight LC neurons (Fig. 1A). To ensure 
only noradrenergic neurons (henceforth LC/NE neurons) were studied, only fluorescence-positive neurons were targeted for recordings in each slice from DBH-Cre: Ai9 mice (Fig. 1A). LC/NE neurons

were further confirmed by

their anatomical location

and stereotypical firing

pattern (see below).

Mature LC/NE neurons at

rest had highly

depolarized membrane

potentials $(-43 \pm 0.14 \mathrm{mV}$,

$\mathrm{n}=289$ ), and all neurons

were spontaneously

active at rest $(1.6 \pm 0.4 \mathrm{~Hz}$,

$n=30$; Fig.1B), similar to

previous reports ${ }^{28-30}$. In

response to negative

current injection, LC/NE

neurons were charged

very slowly (a large Tau)

with a very high input

resistance compared to

classical neurons

(Fig.1C). In response to

prolonged suprathreshold

positive current injection,

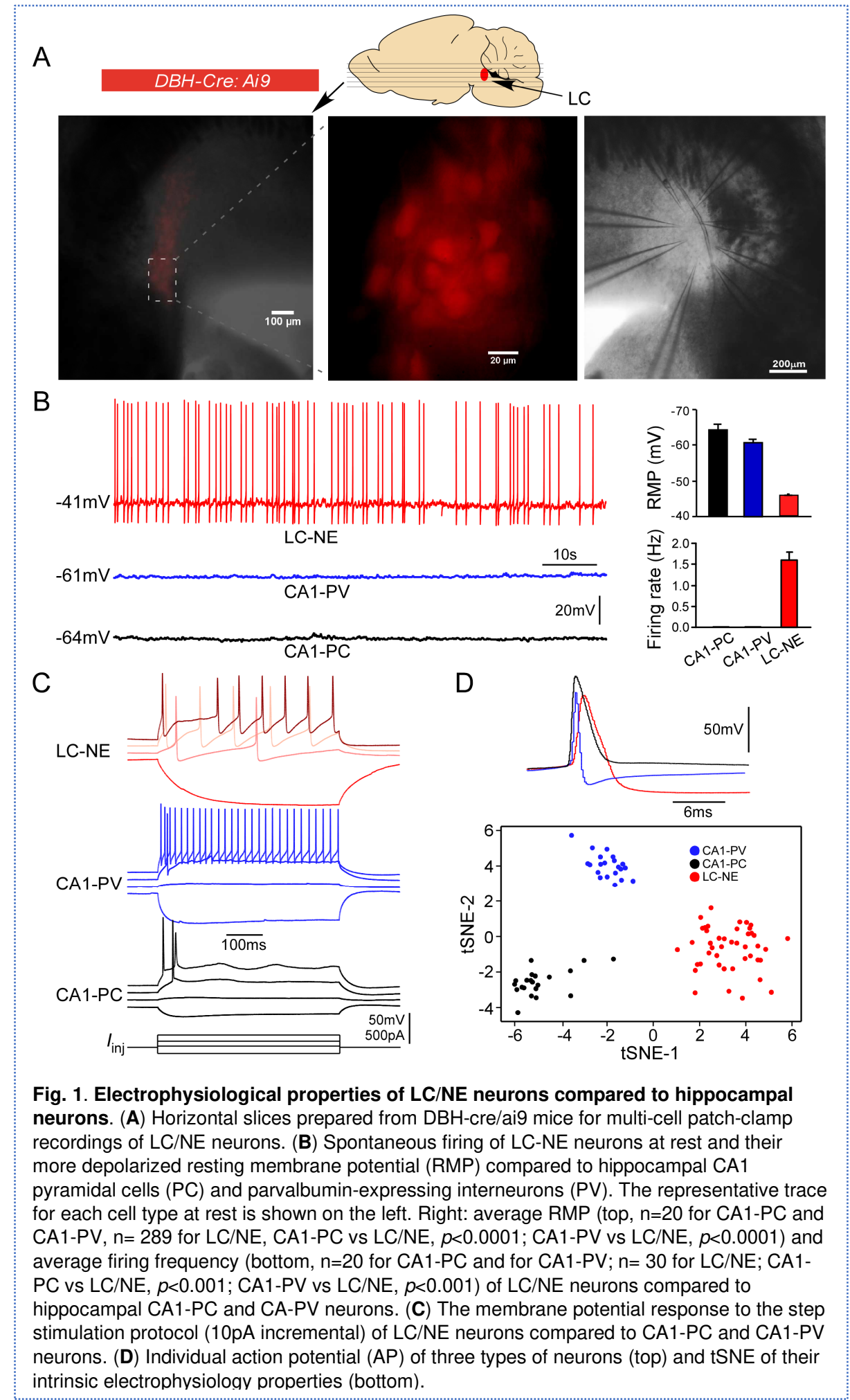

the majority of LC/NE neurons exhibited a characteristic pause after their first action potentials (APs)

followed by a series of APs, which departed significantly from stereotypical firing patterns of classical 
neurons (Fig. 1C). LC/NE neurons were also unique in their single APs, which were much wider with a lower amplitude, followed by a deep and prolonged afterhyperpolarization compared to classical neurons (Fig. 1C, D).

LC/NE cells in our preparation could be recorded stably for at least 1 hour under our recording conditions, suggesting these cells were healthy and the unorthodox intrinsic membrane properties as observed in these neurons were not otherwise due to the technical bias. To further rule out this possibility, we recorded two classical neurons from the hippocampus CA1, pyramidal cells (CA1-PCs) and parvalbumin-expressing fast-spiking interneurons (CA1-PVs), using the same conditions. The resting membrane potentials of CA1-PC and CA1-PV were $-63.9 \pm 1.5 \mathrm{mV}(\mathrm{n}=20)$ and $-60.7 \pm 1.0 \mathrm{mV}$ $(n=20)$ respectively, consistent with those reported in the literature, but significantly different from LC/NE neurons (Fig.1B). In addition, both CA1-PCs and CA1-PVs were barely active at rest (Fig.1B). These direct comparisons indicated unusual electrophysiological properties as observed in LC/NE neurons reflected their real unique cellular phenotypes. To illustrate strikingly distinct properties of LC/NE neurons, we extracted 13 electrophysiological features from three groups of neurons ${ }^{31,32}$ (see Methods, LC-NE, CA1-PC, CA1-PV) and plotted them in tSNE-map. This tSNE-based clustering approach well separated LC neurons from other two groups of classical cortical neurons, highlighting the unique electrophysiological properties of LC/NE neurons (Fig. 1D).

\section{Morphological types of LC/NE neurons in adult mice}

We recovered the morphology of each recorded LC neuron to examine their morphological features (Fig. 2A, 2C, Fig.S1). Most LC neurons were large cells with smooth dendrite (aspiny), similar to CA1PV interneurons in the hippocampus, but different from CA1-PCs with spiny dendrites (Fig.S1). In addition, their dendritic trees were more tortuous with less branch order than hippocampal neurons

(Fig.S1). LC/NE neurons had diverse somatodendritic shapes, based on which we could group them into two major types: fusiform cell (FF) and multipolar cells (MP) following previous terminology ${ }^{33}$. FFs had an elongated, usually spindle-shaped large soma with two, or rarely three, primary dendrites originating from opposite poles of the soma (Fig. 2C, 2D, 2F). The second type of LC/NE neuron, MP, 
had a triangular or nearly round soma with more than three primary dendrites that could originate from

any side of their soma (Fig. 2A,

2B, 2F). The dendrites of MPs

also branched more profusely

quite close to the cell body

compared to FF (Fig.2A,2B;

Fig.S2). To support this LC

neuron classification, we

reconstructed a subset of

LC/NE neurons and quantified

their soma shape (i.e. the ratio

of the major to the minor axis of

a fitted ellipse of the soma) and

dendrite distribution (the

proportion of the dendrites

originated around the major axis

of the fitted ellipse) (Fig. 2E),

and then plotted these two

parameters against each other.

Two groups of LC/NE neurons

were separated in this plot (Fig.

2E). We also trained a non-

linear neural network based

classifier to distinguish cell

types and this classifier could

separate two cell types with a

performance accuracy of $100 \%$ (Fig.S3C). Of note, the performance using soma shape alone was

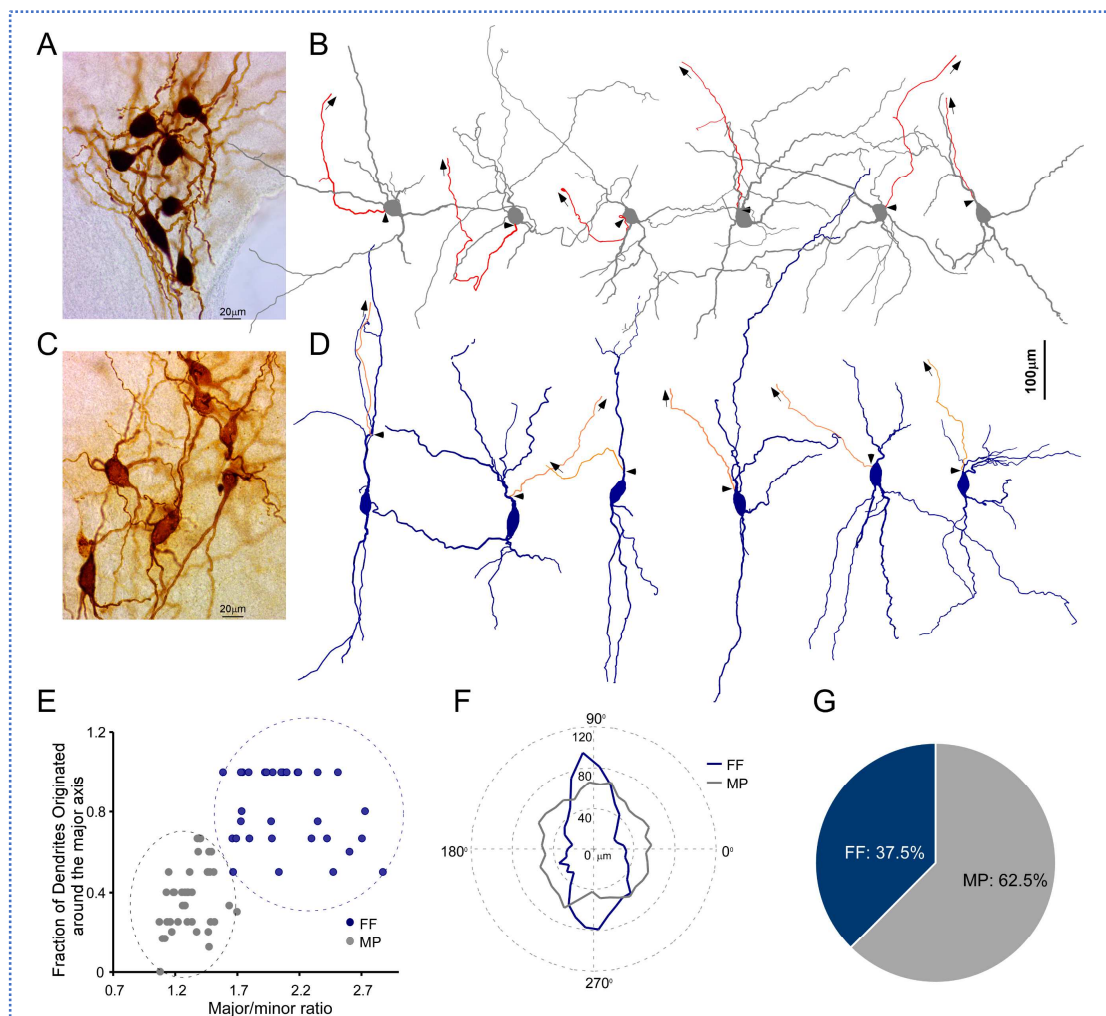

$\mathrm{H}$
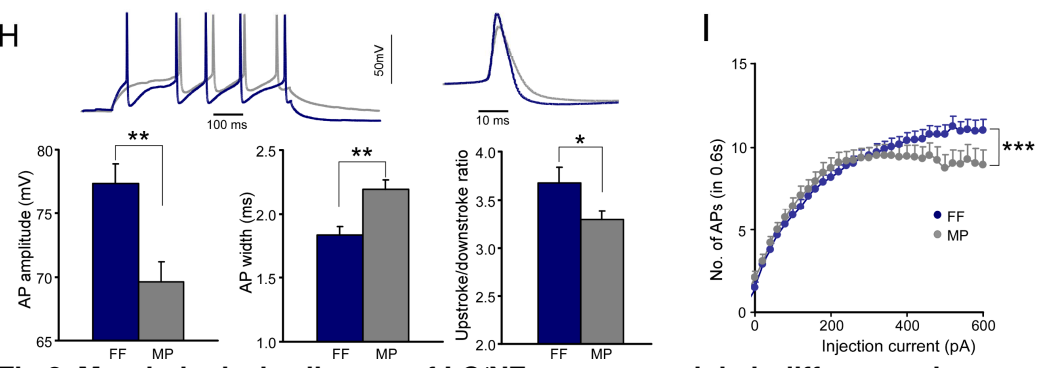

Fig 2. Morphological cell types of $L C / N E$ neurons and their differences in electrophysiological properties. (A) A micrograph shows 7 morphologicallyrecovered LC/NE neurons, most of which are multipolar cells (MP). (B) Morphological reconstruction of 6 MPs. The dendrite and soma shown in gray and the axon shown in red. The axon is shown only partially, and the arrows indicate the trajectory of the major axonal branch. The triangles indicate the origin of the axon. (C) A micrograph shows 7 morphologically-recovered LC/NE neurons, most of which are fusiform cells (FF). (D) Morphological reconstruction of 6 FFs. The dendrite and soma shown in dark blue, and the axon shown in orange. The axon is shown only partially, and the arrows indicate the trajectory of the major axonal branch. The triangles indicate the origin of the axon. (E) Plotting the shape of the soma against the fraction of dendrites originated around the major axis of the soma of each neuron shows the separation of FF and MP ( $n=31$ for FF and $n=39$ for MP). ( $F)$ The dendritic orientation of FF and MP relative to the major axis of their soma. Each morphology is rotated to ensure its major soma axis is aligned with the X-axis $\left(90^{\circ}-270^{\circ}\right)$ in the $2 \mathrm{D}$ space. Zero in the plot indicates the center of the soma ( $n=31$ for FF, $n=39$ for MP). (G) The proportion of each type. (H) Differences in electrophysiological properties between cell types. Top: two representative firing traces in response to suprathreshold depolarizing current injections into MP and FF (left), and individual AP from MP and FF (right). Bottom: Average AP amplitude (left; $F F, n=19 ; M P, n=41 ;{ }^{* *} p<0.01$ ), average AP halfwidth (middle; FF, $\mathrm{n}=19 ; \mathrm{MP}, \mathrm{n}=41 ;{ }^{* *} p<0.01$ ), and Upstroke/downstroke ratio of AP (right; FF, $\mathrm{n}=19 ; \mathrm{MP}, \mathrm{n}=41 ;{ }^{*} p<0.05$ ). (I) The membrane voltage responses to increasing current step injections in FF and MP (FF: $n=39 ; M P, n=37 ; F(1)=29.5$; ${ }^{*} p<0.001$ with two-way ANOVA). 
almost as good as the performance using two parameters (Fig.S3A, B), suggesting soma shape is a reliable criterion to distinguish two types of LC/NE neurons. These analyses supported our manual classification based on the somatodendritic shape.

In addition to somatodendritic differences, two morphological types differed in axonal arborization as well. The majority of LC/NE neurons recorded from our slice preparation had a visible thick axon that originated from either the soma or dendrite (see below), and then arborized away from the fourth ventricle and were inevitably severed due to slicing procedure (as evidenced by a characteristic retraction ball at the end $)^{34}$. FFs generally had the axon originating from one of the primary dendrites ( 95\%, 37 out of 39 reconstructed FF neurons, Fig 2D), while MPs were much more likely to have their axon originating from the soma ( $80 \%, 37$ out of 46 reconstructed MPs; Fig. 2B). In addition, MPs had less local axonal arborization compared to FFs (Fig.S2). The axon in many MPs had the first branching point located $>400 \mu \mathrm{m}$ away from their origin, while the axon in FFs branched much earlier and formed denser local axonal arborization than MPs (Fig. S2).

FFs and MPs had different population sizes (MP: 62\%, 416 out of 666 recovered LC/NE neurons; FF: $\sim 38 \%, 250$ out of 666 LC/NE neurons, Fig.2G). Given that FFs constitute $~ 38 \%$ of total LC/NE neurons (Fig. 2G), the proportion of FFs in each multi-cell recording set (the cohort of $>3$ LCs neurons from each slice) should fall most frequently within the range of $30-40 \%$ if FFs are distributed with the same proportion across the entire LC. However, this was not the case in the histogram of FF proportion (Fig. S4) across all recording sets. Instead, the FF proportions were heavily biased toward either the range of $0-20 \%$ or of $50-100 \%$, suggesting that FFs were not distributed with the same proportion across the entire LC, but preferentially localized in certain anatomical positions. Indeed, we often found individual recording sets in which most recorded LC/NE neurons were FFs (Fig.2C). The same was true when we calculated the proportions of MP (Fig.2A, Fig. S4). This sampling bias might reflect the anatomical preference of different types of LC/NE neurons as reported in the rat $L^{33}$.

To examine if MPs and FFs had different intrinsic membrane properties and firing patterns, we compared 8 electrophysiological parameters between two types (Suppl. Table). FFs had a narrower spike with a larger amplitude than MPs (Fig. 2H, Suppl. Table). The spikes from two cell types also had 
different temporal dynamics (measured at the first spike evoked by step protocol, Fig. 2H, Suppl.

Table). In addition,

FFs could be evoked

more spikes with

large suprathreshold

depolarizing current

injections than MPs

(Fig. 2I).

\section{Chemical}

transmission

\section{between pairs of}

\section{LC/NE neurons}

NE could be released

locally in the LC from

LC/NE neurons, and
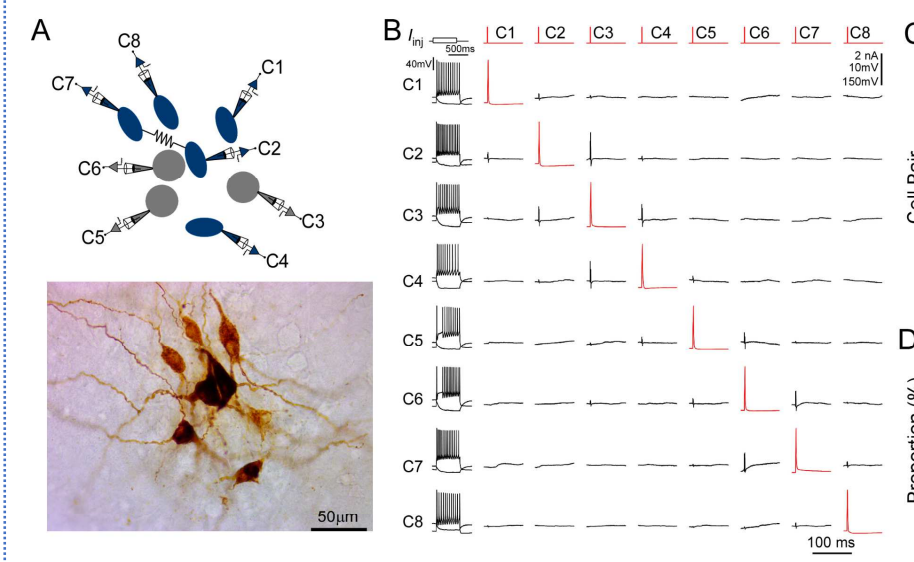

E

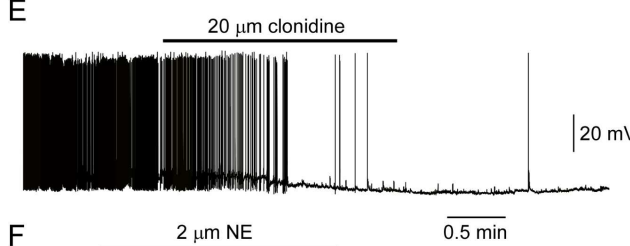

$\mathrm{F}$
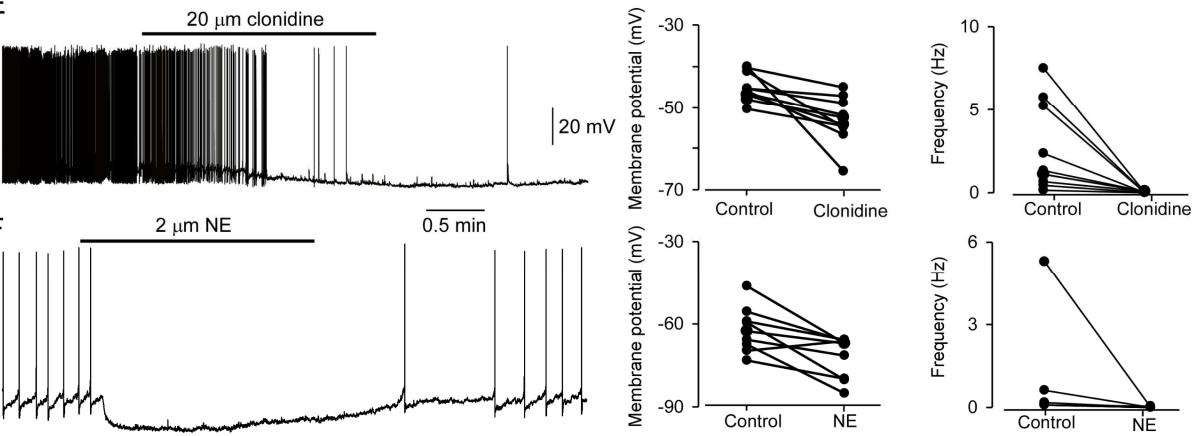

Fig 3. Chemical transmission between LC/NE neurons. (A) Simultaneous patch-clamp recording of eight LC neurons with their morphology post hoc recovered. Cell 7 and Cell 2 is electrically coupled. (B) The membrane response of eight LC neurons to the step protocol (10pA incremental, left) and pairwise testing of synaptic connection (or any other type of interactions) between eight LC neurons. Red traces indicate action potential (AP) evoked in each presynaptic cell and the average traces of the potential postsynaptic responses are shown in black. No prominent membrane hyperpolarization or depolarization following each AP was detected in any cell pair. The traces for electrical coupling (Cell 7 and Cell 2) not shown. (C) Inter-soma distance (ISD) of all tested cell pairs. The majority of cell pairs have an ISD of less than $100 \mu \mathrm{m}$. (D) The connection and nonconnection proportion out of all tested pairs in various conditions. NETI: NE transporter inhibitor. (E) Bath application of clonidine hyperpolarized membrane potentials (Left plot: $n=10, p<0.01$ with paired T-test) and reduced firing frequency of LC neurons at rest (Right plot: $n=10,{ }^{* \star} p<0.01$ with the Wilcoxon-sign rank test). The representative trace is shown on the right. (F) Continuous puffing of norepinephrine (NE) quickly hyperpolarized membrane potentials (Left plot: $n=9, p<0.01$ with paired T-test) and reduced firing frequency (Right plot: $n=9, p<0.01$ with the Wilcoxon-sign rank test) of LC neurons held at -20--40pA. The baseline firing frequency of these cells was much lower due to the negative holding current. The representative trace is shown on the right.

themselves to evoke

hyperpolarization. In addition, LC/NE neurons may co-release glutamate to engage fast

neurotransmission ${ }^{14,15,35}$. To directly test these two connection scenarios, we examined interactions

between any LC/NE cell pair once simultaneous whole-cell recordings of LC neurons were established

(Fig. 3A, B). Single action potentials (APs) or a train of APs were evoked by current injection into

presynaptic neurons, while the membrane potentials of simultaneously-recorded LC neurons were

monitored. An excitatory connection is identified if the excitatory postsynaptic potentials (EPSPs) in

potential postsynaptic neurons are time-locked to presynaptic APs ${ }^{27,31}$. However, with more than 1500 
LC neuron pairs tested (Fig.3A, B, D), no single excitatory connection was detected, despite prominent spontaneous EPSPs recorded in potential postsynaptic neurons. In addition, no visible hyperpolarization in potential postsynaptic cells following single presynaptic APs was detected in these tested pairs as well (Fig.3B), suggesting NE released from single LC neurons was not sufficient enough to reach neighboring cells to activate their a2Ars (NE concentration issue).

Given a wide range of inter-soma distance (ISD) of tested pairs (Fig.3C), we reasoned that we might overcome the potential NE concentration issue by firing a burst of APs (5 APs in $50 \mathrm{~Hz}$ ) in presynaptic LC neurons while potential postsynaptic LC neurons were positioned as close as possible. We tested 708 cell pairs using our bursting protocol, but were still unable to detect any visible hyperpolarization in these cell pairs, including 147 cell pairs with an ISD < 60 $\mu m$ (Fig.3D). In addition, we also examined if we could overcome the concentration issue by incubating slices with NE transporter (NET) inhibitor desipramine $(100 \mu \mathrm{M})$. However, no visible hyperpolarization could be detected in the narrowly spaced cell pairs as well ( $n=62$, Fig.3D). Finally, given that LC/NE neurons were spontaneously active, we reasoned that NE might be depleted from presynaptic cells before the connections were being tested. We thus incubated the slices with another NET inhibitor nisoxetine (1 $\mu \mathrm{M}$ ) in a high $\mathrm{Mg}^{2+} /$ low $\mathrm{Ca}^{2+} \mathrm{ASCF}$ (to prevent the presynaptic release of $\mathrm{NE}$ ) ${ }^{36}$, and then switched to regular ACSF before testing connections. 102 cell pairs with an ISD $<90 \mu \mathrm{m}$ (median: $35 \mu \mathrm{m})$ were tested under this condition, but no hyperpolarization was detected in any cell pair.

To rule out the possibility that $\alpha 2 A R$ may not be significantly expressed in LC neurons of adult animals to initiate hyperpolarization, we bath applied $\alpha 2 \mathrm{AR}$ agonists, clonidine and NE, to examine if these agonists could induce hyperpolarization on LC/NE neurons as expected. Indeed, application of either clonidine or NE could induce hyperpolarization in the majority of LC neurons (Fig.3E, F) while simultaneously suppressing their firing, indicating that a2ARs were indeed expressed in mature LC neurons.

\section{Electrical transmission between pairs of LC/NE neurons}

Our multi-patch recordings also allowed for examining the presence and properties of electrical coupling (EC) between pairs of LC/NE neurons. The presence of electrical coupling was tested by 
recording membrane responses in simultaneously recorded LC/NE neurons following the injection of prolonged depolarizing or hyperpolarizing current pulses $(600 \mathrm{~ms})$ in one of the cells. An example of a coupled pair is illustrated in Figure 4A-C, in which a current pulse in the presynaptic Cell 1 evoked a membrane

response of the

same sign in the postsynaptic Cell

4 , although of

much lower

amplitude. The

same occurred

when we tested

the other way

around. Other

tested pairs did not

show evidence of

EC in either

direction (Fig. 4C).

The steady-

state coupling

coefficient (CC),

defined as the

voltage change at

the postsynaptic
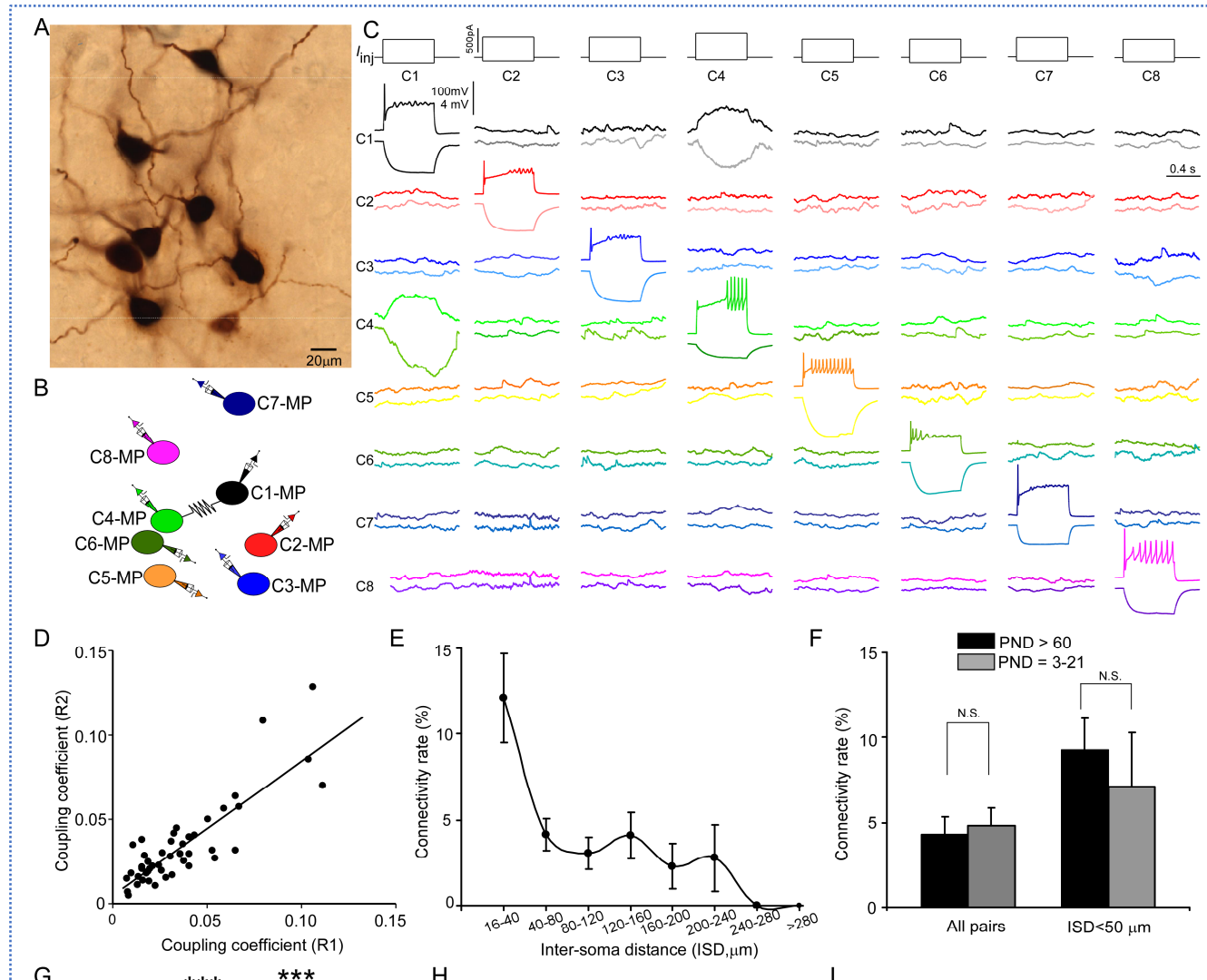

G
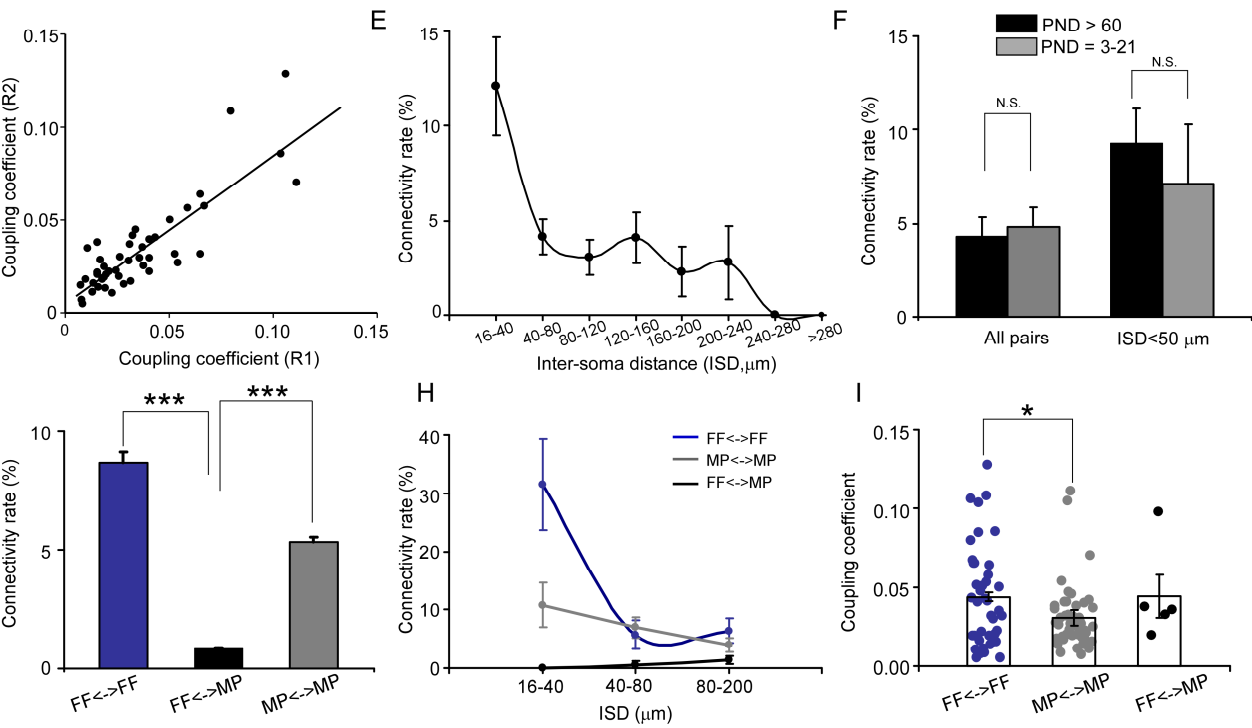

Fig. 4. Electrical coupling between LC/NE neurons. (A) A micrograph shows 8 morphologicallyrecovered LC/NE neurons. (B) The relative location of eight recorded neurons (with assigned number) depicted as above and their connections. (C) Pairwise testing in the 8-cell recording set as shown in $\mathbf{A}$ reveals electrical coupling between Cell 1 and Cell 4. No sign of EC between the rest of cell pairs. (D) Estimates of the coupling coefficient (CC) in both directions (Cell 1 to Cell 2, and Cell 2 to Cell 1) are plotted against each other, indicating that most contacts lack significant rectification. The data are fitted with a straight-line function $\left(\mathrm{R}^{2}=0.75, p<0.001\right)$. (E) The EC connectivity rate as a function of the intersoma distance (ISD). (F) The EC connectivity rate as a function of age. N.S.: no significant difference. (G) The EC connectivity rate as a function of cell type across all cell pairs. ${ }^{* * *} p<0.0001$. (H) The EC connectivity rate as a function of cell type within the same ranges of ISDs. (I) The CCs in two homotypic types of ECs (among the same cell type) and one heterotypic type of EC (among the different cell types). ${ }^{*} p<0.05$.

cell divided by the voltage change at the presynaptic cell measured at the last $10-20$ ms period of the current step (see Methods), was calculated for each recorded pair. A pair of LC/NE neurons was 
the noise of the recording conditions ${ }^{37}$. According to this criterion, 65 of the 1503 tested pairs across the ISD range of 16-531 $\mu \mathrm{m}$ were electrically coupled (4.3\%; Fig. 4F, also see below). For each coupled pair, the CCs were estimated and expressed as the average of the values in both directions. The steady-state CC averaged $0.040 \pm 0.028$ (SD) (range: $0.0051-0.13 ; n=65)$ (Fig.4D, I). The strength of electrical transmission in the vast majority of coupled pairs was bidirectional and symmetric (Fig.4C, D). Estimates of CC in both directions for each pair showed a positive correlation with a slope of 1.03 (Fig. 4A), not significantly different from 1 ( $p=0.8$; Fig. 4D), indicating that gap junctional conductance between LC/NE neurons was largely nonrectifying.

Electrical coupling generally occurs between narrowly-spaced cell pairs. Given the wide range of ISDs of tested pairs, a low coupling rate in our dataset was not unexpected. To examine the spatial profile of EC in LC/NE neurons, we measured the ISDs of all tested pairs, including coupled pairs and uncoupled pairs. Cell pairs were much more likely to form gap junctions (GJ) when their ISDs were less than $40 \mu \mathrm{m}$. The coupling rate dropped quickly as the ISD increased from $40 \mu \mathrm{m}$ (Fig.4E). With the ISD $<40 \mu \mathrm{m}$, the coupling rate between LC neurons was $\sim 12 \%$, indicating GJs were prominent in adult mice. Interestingly, while the coupling rate was much lower in cell pairs with the ISD $>40 \mu \mathrm{m}$, it was much more resistant to the increase in the ISD (Fig. 4E). The coupling rate remained relatively constant when the ISD increased from $40 \mu \mathrm{m}$ to $240 \mu \mathrm{m}$ before dropping to zero, with a second small peak of the rate occurring around $140 \mu \mathrm{m}$.

Several previous reports suggest gap junctions among LC/NE neurons decline significantly with age $^{20-22}$. To examine if this is the case, we performed multi-cell recordings of LC neurons from mice at the age of PND 13-21 (Fig. 4F), expecting a much higher coupling rate. Surprisingly, the coupling rate at this young age was very similar to adult age $(4.8 \% ; 8$ connections out of $165, p>0.05$, Fig. $4 \mathrm{~F})$. The coupling rate was still very similar between the two ages when the ISD was controlled (Fig. 4F).

\section{Electrical transmission by morphological cell type in LC}

Electrical coupling generally occurs between cell pairs of the same cell type in many brain regions ${ }^{38,39}$. Given that LC/NE neurons were classified into two major morphological cell types (MP and FF), we thus set to examine if EC between LC/NE neurons was cell-type specific. We examined all tested pairs 
that had their morphology recovered and assigned each cell into either FF or MP using the same criteria (somatodendritic shape), and then examined the coupling rate as a function of cell type (Fig.36). Of all FF-FF pairs (both cells are FF in a pair), we found $8.7 \%$ were electrically coupled (Fig. 4G); of all MP-MP pairs (both cells are MP in a pair), 5.3\% were electrically coupled (Fig. 4G). By contrast, only $0.8 \%$ of FF-MP pairs (two cells are FF and MP in a pair) were coupled (Fig. 4G; $p<0.0001$ for both comparisons). While the coupling rate for FF-FF pairs was slightly higher than that for MP-MP pairs, there was no significant difference between these two $(p=0.08)$. Given the coupling rate is sensitive to the ISD, we then compared their rates within the same ISD range to rule out the possibility that the rate differences we observed were due to the differences in ISD. Interestingly, with the ISD of up to $40 \mu \mathrm{m}$, no connection between FF-MP pairs was identified ( 0 out of 55 tested pairs), while the rates for those FF-FF and MP-MP pairs were much higher (11 out of 35 tested pairs, $31.4 \%$ for FF-FF, $p<0.01$ compared with MP-FF; 7 out of $65,10.7 \%$ for MP-MP, $p<0.01$ compared with MP-FF; Fig. 4H). The coupling rate for FF-FF pairs was also significantly higher than for MP-MP pairs $(p=0.01$, Fig. $4 \mathrm{H})$. With the ISD range of $40-80 \mu \mathrm{m}$, the coupling rates for both MP-MP and FF-FF pairs were also significantly higher than that for FF-MP pairs ( $p<0.01$ for both comparisons, Fig. $4 \mathrm{H}$ ), but there was no significant difference between the MP-MP coupling rate and FF-FF coupling rate $(p=0.67)$. EC in MP-FF pairs most occurred in the cell pairs with the ISD $>80 \mu \mathrm{m}$, but their rate in this ISD range was still lower than MP-MP and FF-FF pairs (FF-FF vs FF-MP: $6.3 \%$ vs $1.4 \%, p<0.01$; MP-MP vs FF-MP: $3.9 \%$ vs $1.4 \%$, $p=0.05$, Fig. $4 \mathrm{H}$ ). These results indicate that electrical connections in LC neurons generally form among cell pairs of the same cell type (Fig.3-6). In addition, FFs more likely connect with FF than MPs connect with MPs, particularly if they are narrowly spaced. In addition, EC in FF-FF pairs had higher connection strength (higher coupling coefficients (CCs)) than EC in MP-MP pairs (Fig. 4I).

\section{Putative electrical synaptic location in LC}

We reason that electrical synapses between FFs (FF-FF) and between MPs (MP-MP) may locate in different somatodendritic domains (soma vs dendrite), which may contribute to their CC difference. The majority of recorded neurons have sufficient morphology quality allowing for examining the putative synapse in those coupled pairs. We traced the entire somatodendritic domain of the coupled pair under 
one cell merged

with a dendritic

segment from

another cell at one

or multiple crossing

points (Fig. 5A-C),

while we barely

found this close

contacts in

uncoupled cell

pairs. We thus

believed these

contacts were

electrical synapses

and set this

standard to identify

the putative

electrical synapses

among coupled

pairs.

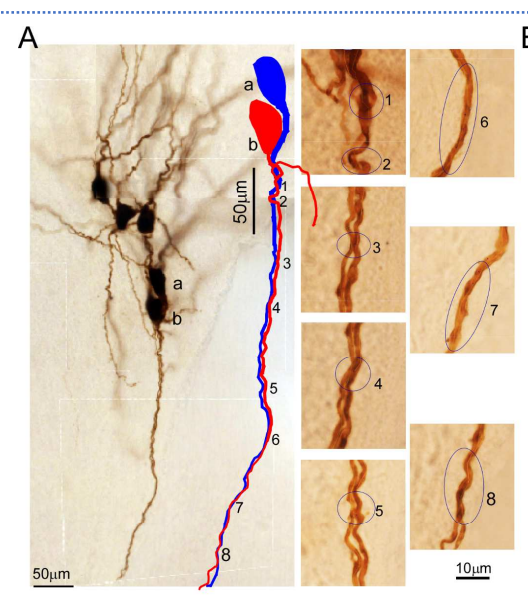

$\mathrm{D}$

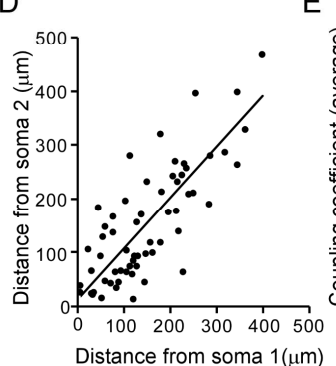

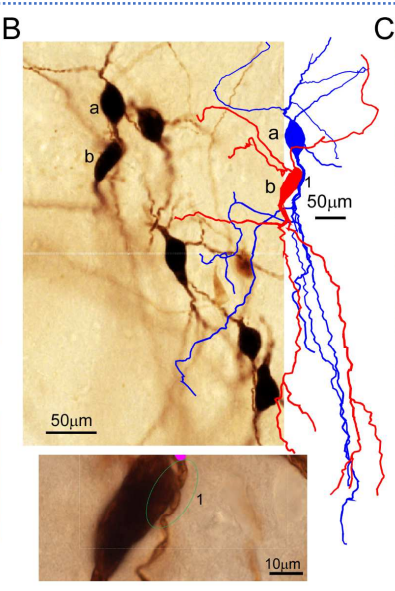

$\mathrm{F}$

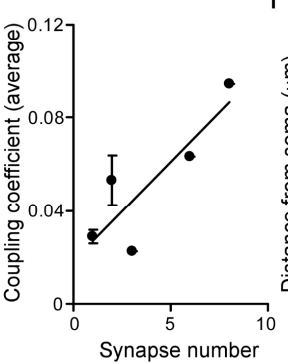

C

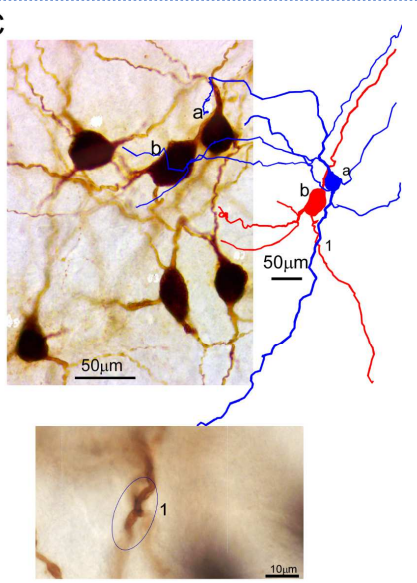

G

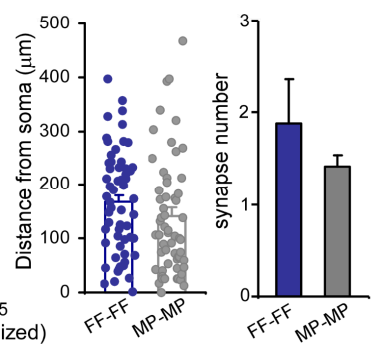

Fig.5 Gap junctions (GJ) between LC/NE neurons. (A) Left: A microphotograph depicting 6 morphologically recovered LC/NE neurons (simultaneously recorded), among which Cell a and Cell b were identified as a GJ-coupled pair. Multiple putative dendrodendritic electrical synapses can be identified after their morphology was reconstructed (as labeled 1 from 8). Right: high-magnification view of each putative electrical synapse as shown on the left. (B) Top: A microphotograph depicting 7 morphologically recovered LC/NE neurons, among which Cell a and Cell b were identified as a GJcoupled pair. A putative somatodendritic electrical synapse was identified after their morphology was reconstructed (as labeled as 1). Bottom: high-magnification view of the putative electrical synapse as shown on the top. (C) Top: A microphotograph depicting 6 morphologically recovered LC/NE neurons, among which Cell a and Cell b were identified as a GJ-coupled pair. A putative dendrodendritic electrical synapse was identified after their morphology was reconstructed (as labeled as 1). Bottom: highmagnification view of this putative electrical synapse as shown on the top. (D) Dendritic distances from the electrical synapse to Soma 1 are plotted against the distance from soma 2 for each putative electrical synapse. Correlation coefficient $r=0.79, p<0.01$. The slope of the straight line is 0.66 . (E) The putative synapse number was plotted against the $C C$ for each GJ-coupled pair. $r=0.86, p<0.05$. (F) The distances from each putative electrical synapse to two somata were averaged and then plotted against the $\mathrm{CC}$ of each GJ-coupled pair. The CC was normalized if a GJ-coupled pair had multiple putative synapses. $r=$ 0.19. (G) Comparing the synapse number and the dendritic distance away from the soma of putative electrical synapses for two homotypic EC types (FF-FF vs MP-MP: $p=0.19$ in the dendritic distance; $p=0.43$ in the synapse number).

For many coupled cell pairs, only one putative synapse could be identified (Fig. 5C). More than 2 putative synaptic contacts could be identified in some coupled pairs as well (Fig. 5A), which were more frequent in FF-FF pairs (Fig. 5G). This may be one reason for a higher connection strength in FF-FF coupled pairs compared to MP-MP coupled pairs. Indeed, plotting putative synaptic numbers against 
synapses were found to form between dendrites. This could be a single crossing point between two dendrites from a coupled pair (Fig.5C) or an entire dendrite from one cell could position in the perfect parallel with the entire dendrite from another cell, and they merged several times (form multiple putative synapses) along their course to the terminals (Fig. 5A). Only 2 coupled pairs (one FF-FF and one MPMP coupled pair) had putative synapses formed between dendrite and soma (Fig.5B). No putative synapses formed between two somata were identified in our data. Therefore, there was no preferential synaptic location for either FF-FF coupled pairs or MP-MP coupled pairs.

The dendritic branches where putative synapse forms were generally first or second-order branches and the synapses were most frequently located in the middle of the entire dendritic tree (average dendritic distance from soma: $155 \mu \mathrm{m}$, Fig.5G). When one cell pair has multiple contacts, these contacts were generally localized in the same major dendrite or the same dendritic branch of the same order of each cell. For each putative synapse, the dendric distance away from one soma was linearly correlated with the distance from another soma with an $\mathrm{R}$ of 0.86 , indicating the synapses sit with a similar dendritic distance away from two somata of the coupled pair (Fig. 5D). The average dendritic distance of putative synapses for FF-FF pairs was $168 \mu \mathrm{m}$, tending to be slightly greater than the distance for MP-MP pairs (140.0 $\mu \mathrm{m}$, Fig. 5G). In addition, the dendritic distance of the putative synapse appeared to negatively correlate with CCs (Fig. 5F).

\section{Connectivity patterns of electrical coupling beyond two LC neurons}

Multi-cell recordings also allowed for examining electrical coupling beyond two cells. In many individual recording sets, we could identify multiple electrical connections among a cohort of cells. An example of such a case was illustrated in Figure 6A-C, in which a current pulse in the presynaptic Cell 6 evoked a membrane response of the same sign in postsynaptic Cell 1 and 8 . The current pulse in the presynaptic Cell 1 or 8 evoked a membrane response of the same sign in postsynaptic Cell 6 , but not 8 or 1 respectively. This indicates Cell 1 and Cell 8 were both electrically connected to Cell 6 , but they were not directly connected to each other. This result also indicates that electrical signals from one cell hardly pass to other cells via second-order coupling if they are not electrically coupled in the first order. Other tested pairs did not show evidence of electrical coupling in either direction (Fig. 6C). 
We had 15 individual recording sets from which more than two electrical connections (or GJcoupled pairs) were identified, and three of them have three connections identified. In these recording sets with multiple coupled pairs identified, we found a coupled pair often shared the same coupling partner with other coupled pairs as illustrated in Fig $6 \mathrm{~A}-\mathrm{C}(\mathrm{A} \leftrightarrow \mathrm{B} \leftrightarrow \mathrm{C} ;$ each letter representing a cell. Fig. 6D). This is surprising since two coupled pairs should have been less likely to share the same partner if
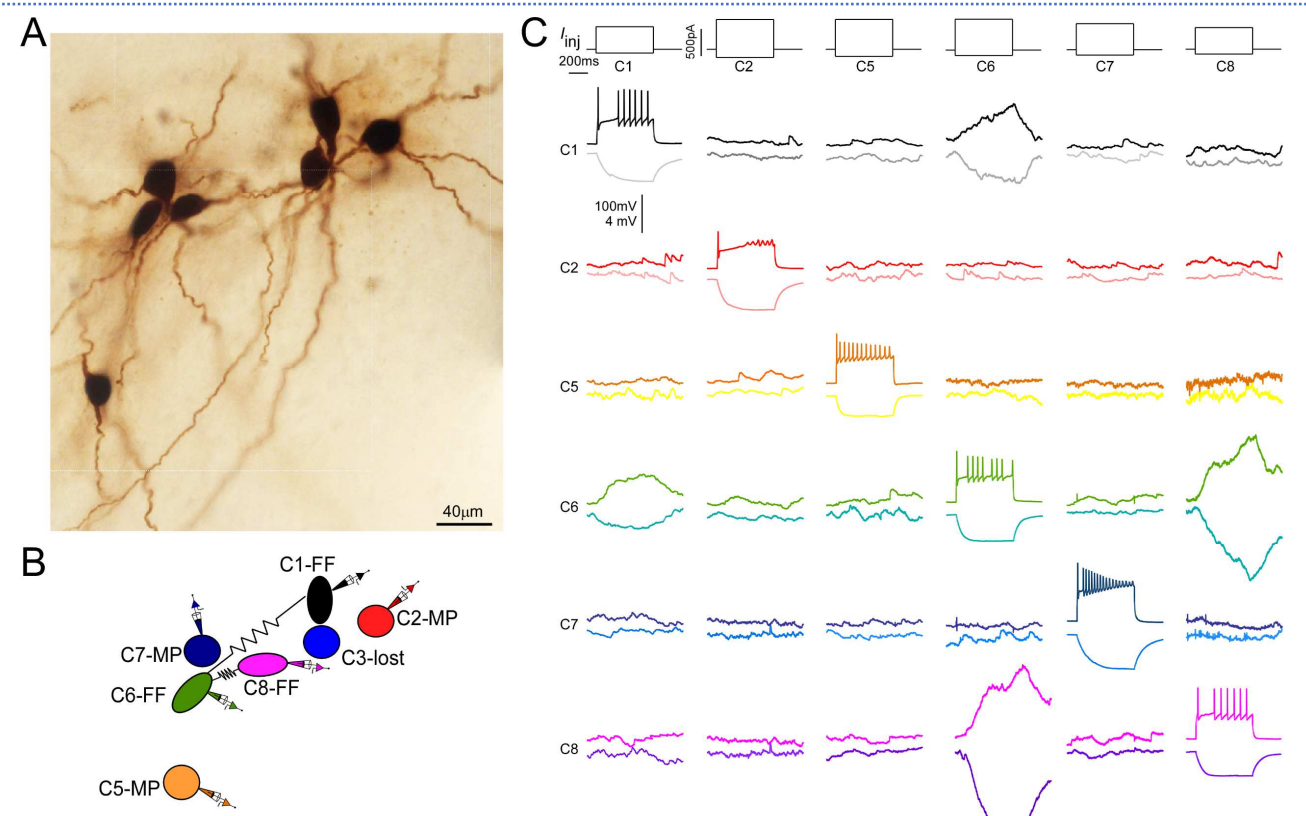
$100 \mathrm{mv}$
$4 \mathrm{mV}$

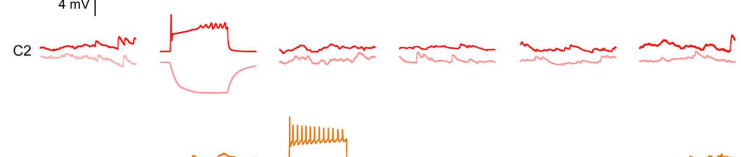
C5
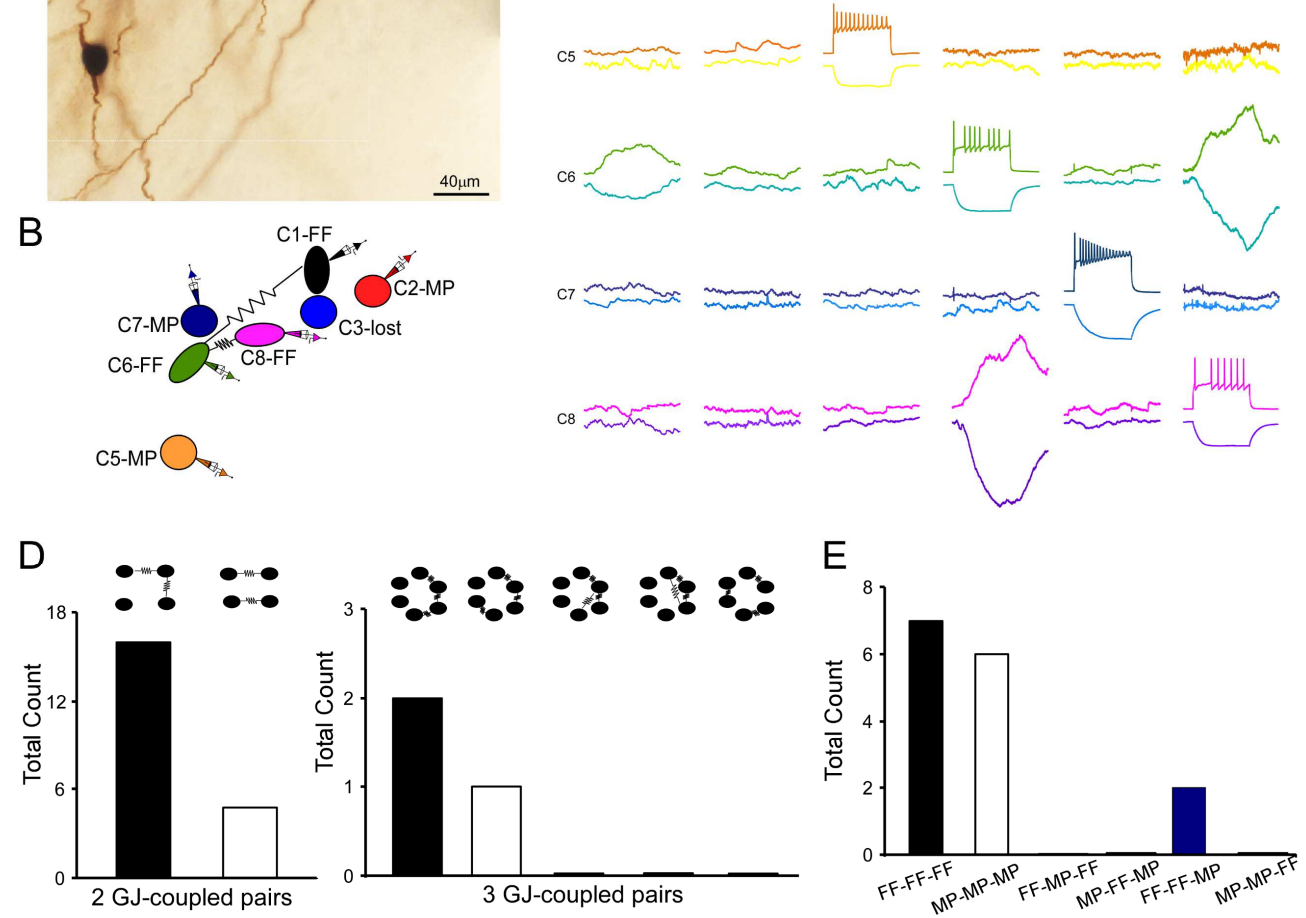

Fig.6. Multiple GJ-coupled pairs identified in a cohort of LC/NE neurons. (A) A micrograph shows six morphologically-recovered LC/NE neurons (simultaneously recorded). (B) The relative location of six recorded neurons (with assigned number) depicted as above and their connections. (C) Pairwise testing in the 6-cell recording set as shown in A reveals electrical coupling (EC) between Cell 1 and Cell 6, and between Cell 6 and Cell 8. No sign of EC between the rest of cell pairs. (D) The counts of multiple GJcoupled pairs identified in individual multi-cell recording sets with all possible arranging patterns (top). (E) The cell-type identity of each cell in those sequentially-connected GJ-coupled pairs presented in $D$, and the frequency for each possible combination.

each cell is equally coupled with other cells in the network at a certain rate. However, two coupled pairs that do not share the partner $(A \leftrightarrow B / C \leftrightarrow D)$ were rare in our recording sets (Fig. 6D). These data suggest each cell is not equally coupled with other cells in the network, instead, GJ-coupled cells tend to cluster together and form a train-like pattern $(A \leftrightarrow B \leftrightarrow C)$. To test this hypothesis, we started by assessing how well a randomly connected network describes our dataset ${ }^{40}$. In this model, the existence of an electrical connection between any two neurons was independently chosen with a uniform probability $\mathrm{p}(0.0438,73$ out of 1668 pairs tested), which we obtained from the overall pairwise 
connectivity rate across all of our recordings. We first tested this random connectivity model in predicting the patterns of two electrical connections in our recording sets ignoring cell type information (Model 1). Specifically, based on random pairwise connectivity ignoring cell types, we calculated expected numbers of a train-like pattern of two connections $(A \leftrightarrow B \leftrightarrow C)$ versus a non-train-like pattern of two connections $(A \leftrightarrow B / C \leftrightarrow D)$ using our recording sets of 3-8 neurons. We found that the observed number of the train-like pattern ( $n=16$, Fig.6D) was higher than the numbers predicted by the random connectivity model $(n=12.9,95 \%$ confidence interval $(C . I)=[6,21])$, whereas the observed number of the non-train-like pattern ( $n=5$, Fig.6D) was significantly less than the numbers predicted by the model

$(n=11.6,95 \%$ C.I $=$

$[6,19], p=0.01)$. We

calculated the ratio

of the train-like

pattern number to

the non-train-like

pattern number as

a measure of how

electrical

connections to

cluster, which we

henceforth called

cluster index (Fig.

7A). The cluster

index in our dataset was 3.2, which was significantly higher than the number predicted by the model

$(1.1, p=0.001)$. We then tested if differences in the pattern of two connections (observed vs. predicted) can be better explained by cell type-specific connectivity rule as we deciphered ((see Fig.4G) by incorporating cell type information into the model. That is to say, pairwise connection probabilities for FF-FF, FF-MP, and MP-MP pairs (see Fig.4G) were used to obtain a new model prediction. With this 
revised model (Model 2), the observed numbers on average of the train-like pattern still remained higher than the predicted numbers $(n=12.6,95 \%$ C.I. $=[7,18])$, and the observed numbers of the nontrain-like pattern were still significantly lower than the number $(n=11.2,95 \%$ C.I. $=[6,16], p<0.001)$ predicted by the model (Fig. 7B). The cluster index predicted by Model 2 was very similar to that predicted by Model 1, and significantly lower than the observed index (Fig.7A), indicating that the cell type-specific rule can not explain our dataset. Given that neurons of the same type tended to be near

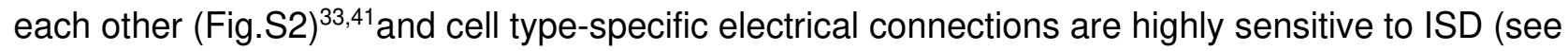
Fig.4H), we further revised our model (Model 3) by zeroing out connections between cell pairs that were greater than $80 \mu \mathrm{m}$, and by using the FF-FF and MP-MP connection rate for cell pairs within 40/80 $\mu \mathrm{m}$ apart and FF-MP connection rate for cell pairs within $80 \mu \mathrm{m}$ apart. The results did not fundamentally change: the observed number of the train-like pattern was not significantly different from the predicted value $(n=19.4)$, but the observed number of the non-train-like pattern $(n=4)$ was still significantly lower than that $(n=15.9)$ predicted by the model. The cluster index predicted by Model 3 was still significantly lower than the observed index (Fig.7A), indicating additional spatial connectivity rules could not explain our dataset as well. In summary, the clustering of two connections in our dataset (a train-like pattern) can not be explained by the random model and spatial and cell type-specific rule as shown above, suggesting an additional, as yet unknown rule underlying the organization of electrical connections in the LC network (Note that all three models shared the assumption that the probability for two neurons to form a connection is independent of all other pairwise connections, an assumption that the new rule will perhaps have to violate). Such an underlying principle could impose on both cell types, and thus the most frequent cases of two connections for multiple coupled pairs are FF-FF-FF and MPMP-MP, while other combinations are very rare or not observed (Fig. 6E).

Frequent observation of the train-like organization of two electrical connections suggests that a GJ-coupled neuronal network may be wired as a train-like network structure (Fig. 7C). Indeed, when we examined the patterns of three electrical connections in those individual recording sets, the most frequent was still the train-like pattern $(A \leftrightarrow B \leftrightarrow C \leftrightarrow D$, Fig. 6D), while other possible organizational patterns including circular-like and hub cell-like patterns were barely observed in our dataset (Fig. 6D). 
We then extended our models to examine three coupled pairs. Our random connectivity model (Model 1) predicted 2.0 train-like patterns of triple connections on average $(95 \%$ C.I. $=[0,6])$ across all our recordings which was the same as the actual observed number, while it predicted 3.0 non-train-like triple connections on average $(95 \%$ C.I. $=[0,7])$, which was greater than the observed number $(1)$. We again used the ratio of train-like to non-train-like patterns to measure the train-like tendency in our dataset and compare it with model predictions. The observed ratio in our dataset was 2 , significantly higher than the predicted ratio $(0.68 \pm 0.25, p=0.001$, Fig.7B). Our Model 2 (see above) also predicted 2.2 train-like pattern of triple connections across all our recording sets on average $(95 \%$ C.I. $=[0,6])$; however, it predicted 4.9 non-train-like triple connections $(95 \%$ C.I. = $[2,8])$ which was significantly greater than the observed number $(n=1, p=0.01)$. Again, the observed ratio was significantly higher than the ratio predicted by Model $2(0.52 \pm 0.47, p<0.05$, Fig.7B). Similar results were obtained using our Model 3 (see above, incorporating inter-neuronal distance), which predicted 6.8 of the train-like patterns of triple connections on average and 9.3 non-train-like triple connections on average. Once again, the observed ratio was significantly higher than the ratio predicted by the third and final model $(0.76 \pm 0.24$, $p<0.001$, Fig.7B). All simulations indicate that, in addition to spatial and cell type-specific rule, an additional train-like rule applies for the organization of three electrical connections.

\section{Discussion}

In this study, we overcame the technical barriers and adopted an 8-patch recording system into adult brainstem slices to record up to 8 noradrenergic neurons simultaneously in the locus coeruleus (LC). Multi-cell simultaneous patch-clamp recordings (more than 2 cells) have been developed to study cortical circuits for a while, but it remains a challenge to apply this technique to small nuclei in the brainstem such as the LC given the space restraint and limited cell number in each brain slice. By optimizing the slice quality and adapting our multi-patching system to small brainstem slices, we could routinely record mature LC/NE neurons simultaneously in the cohort of an average of 6 cells. This technical development allowed for interrogating local LC circuit organization, profiling cellular diversity of LC/NE neurons while simultaneously mapping their connections at a single-cell resolution. In fact, 
this study may represent the first multi-cell patch recordings of a monoamine neuromodulatory system in the brain, allowing for examining how a monoamine released from an individual neuron affects other neurons at a single-cell resolution. Our results indicate LC/NE neurons could be classified into at least two major morpho-electric cell types, and each cell type may form discrete network modules via gap junctions (GJs). Given these cell types may have preferential locations and different projection targets, each GJ-coupled subnetwork may synergize their output as a whole to engage distinct functions of the circuits within its efferent domain. In addition, our large dataset has allowed us to test several longstanding hypotheses regarding local circuit interaction in LC, including a2AR-mediated actions and glutaminergic synaptic connection.

\section{Morpho-electric diversity of LC/NE neurons}

Morphological heterogeneity of LC neurons has been noticed since the very early LC studies. The ventral and anterior part of the LC appears to be dominated by the cells with the stellate multipolar soma shape, while the dorsal part is dominated by the cells with bi-tufted fusiform soma shape ${ }^{33,41}$. In addition, these morphologically distinct LC neurons preferentially project to different brain areas ${ }^{41,42}$, warranting further exploration of the functional correlate of their morphological diversity. To this end, we recovered the morphology of a large number of LC/NE neurons via biocytin staining to characterize their morphological features, providing a quantitative, less ambiguous classification of LC/NE neurons. With their detailed morphology recovered, we confirmed that the soma shape of LC/NC neurons could be either fusiform, round, or pyramid ${ }^{33,41}$. Cells with the fusiform soma shape have a polarized dendritic arborization (bitufted or bipolar), while cells with round or pyramid soma shape have much less polarized dendrite arborization, giving rise to multipolar somatodendritic shape revealed by previous histological staining. This difference in soma shape and dendritic polarization of LC/NE neurons allowed for classifying LC neurons into two morphological cell types - multipolar cell (MP) and fusiform cell (FF) following earlier terminology. This classification was confirmed by a machine learning algorithm. To further support this classification, we found that MP and FF cells, in addition to having distinct somatodendritic shapes, have different local axonal arborization. In addition, MPs and FFs have distinct intrinsic electrophysiological properties. While mature LC/NE neurons as a whole exhibit the 
stereotypical physiological properties that clearly differentiate them from classical neurons, there are subtle differences between MP and FF in their individual action potentials (APs): MPs had a longer AP with a smaller amplitude than FFs. Furthermore, FFs were more likely than MPs to sustain highfrequency firing with large suprathreshold depolarizing current injections. The heterogeneity in AP shape across cell types may be consistent with "narrow" or "wide" units identified during extracellular recordings of rat $L^{11}$. While caution should be taken in comparing intracellular and extracellular waveforms, we speculate that narrow units may come from FFs while wide units come from MPs. If these correspondences can be established, it would significantly facilitate the study of LC-NE neuron ensemble physiology through extracellular recordings in vivo ${ }^{11,43}$.

The differences in morphology and electrophysiology between two cell types suggest they may participate in distinct functions of the circuits within their efferent domains. Indeed, previous studies indicate cells with fusiform soma shape in the dorsal horn preferentially project to the neocortex and hippocampus, while the neurons with multipolar soma shape in the ventral horn preferentially project to the spinal cord and cerebellum ${ }^{41,42}$. While it is difficult to relate each cell in our slice recordings to their anatomical location in vivo, overrepresentation (or underrepresentation) of either FFs or MPs in individual recording sets suggests the anatomical preference of different types of LC/NE neurons in vivo. FF cells as we defined here thus may be recorded from the dorsal horn corresponding to those cells with fusiform soma shape, while MP cells may correspond to those multipolar cells in the ventral horn. If this is true, these two subtypes as we defined here might form separate LC subnetworks with distinct projection domains, each performing a non-overlapping function in the brain (also see below).

We group LC/NE neurons into two major cell types based on morphology. While each of these cell types can be further classified into subtypes, our large-scale characterization of LC/NE neurons constitutes an important step toward understanding their heterogeneity and paves the way for future exploration of their genetic basis and functional relevance. To this end, the recently emerging new techniques such as single-cell RNA-sequencing could be applied to the LC to profile the transcriptomic heterogeneity of NE neurons ${ }^{32,44,45}$. With this approach, identifying the marker genes that differentiate FF from MP subpopulations will pave the way to assessing the unique projection targets and functions 
of circuits within the efferent domain of each cell type. For instance, one can leverage an intersectional genetic strategy using Dbh (encoding dopamine beta-hydroxylase, labeling all NE neurons) and an additional identified type-specific marker to label and manipulate each cell type while animals are trained to perform behavioral tasks.

\section{Chemical transmission between LC/NE neurons}

The physiologic function of monoamine neurons is generally considered as being modulatory based upon the fact that most monoamine axon terminals in the CNS are "asynaptic" free nerve endings thought to mediate "volume transmission" and to modulate the activity of nearby fast-acting synapses releasing glutamate or GABA. However, many lines of evidence suggest monoamine axon terminals, including noradrenergic terminals, co-release glutamate. At least a subset of their axonal terminals makes the junctional or conventional-looking synaptic contacts onto targeted neurons, possibly mediating rapid glutamatergic synaptic transmission ${ }^{13-15,46}$. While evidence for this emerging concept of monoamine function is pretty convincing ${ }^{15}$, a direct demonstration of the glutamatergic nature of synapses established by monoamine neurons is still lacking and simultaneous recordings from single monoamine neurons pose an ideal approach to provide decisive evidence in this regard. We thus took advantage of our unprecedented multi-cell patching recordings to examine if there is any glutamatergic synaptic transmission between LC/NE neurons. Given the high-throughput nature of our system, we were able to test more than 1,500 cell pairs, including those cell pairs spaced very narrowly $(<40 \mu \mathrm{m}$ apart). However, we did not find evidence for glutamatergic synaptic transmission between LC/NE neurons. Nevertheless, this does not rule out the possibility that a subset of noradrenergic axonal terminals does make glutamatergic synapses onto other target regions, including the spinal cord and the parabrachial nucleus neurons ${ }^{13-15}$. Given that only a small subset of all terminals established by monoamine neurons may actually be specialized for the synaptic release of glutamate ${ }^{46}$, those local axon terminals from LC/NE neurons may be either pure noradrenergic or never make any conventional synaptic contact with neighboring noradrenergic neurons. The glutamate co-transmission thus may not apply to the local connection of LC/NE neurons. 
Monoamine release is controlled by their autoreceptors in the presynaptic terminals. These same receptors are often expressed in the somatodendritic domains of monoamine neurons, activation of which increases potassium conductance (membrane hyperpolarization) as seen in their terminals. Alpha2 adrenoceptors ( $\alpha 2 \mathrm{AR})$ are such autoreceptors for NE and are expressed in the somata of LC/NE neurons as well. While a2AR-mediated action on LC neurons is well established, it is still unclear under what conditions these receptors are activated and what is their natural agonist. These receptors may be expressed in the soma to sense NE locally released from other or the same LC/NE neurons, and/or epinephrine released from the medulla oblongata ${ }^{47,48}$. It appears that NE could be released locally from local axonal terminals or somatodendritic domains of LC neurons following a single AP, but it is unknown if NE released from individual neurons is sufficient enough to activate a2AR to evoke the subsequent effect. After testing more than 1500 cells pairs under different conditions, we did not find any detectable hyperpolarization following the stimulation of a single LC/NE neuron. While many other unknown reasons may contribute to this observation, the most plausible explanation is that NE released from individual LC/NE neurons is not sufficient to activate $\alpha 2 A R$ to evoke a visible effect on LC/NE neurons at least in our in vitro preparation. The a2AR-mediated effect may be more like a population-wide phenomenon, only in play when many neurons are simultaneously activated. Alternatively, the NE release from its dense core vesicles may require a specific firing mode, and sufficient NE is released from individual LC/NE neurons only when they fire at a high-frequency, unique firing mode ${ }^{19}$. Which mechanism contributes to the absence of $\alpha 2 A R$-mediated effect in our slice preparation warrants further investigations.

\section{Electrical connection between LC/NE neurons}

Previous studies suggest electrical coupling (EC) in the LC may be negligible or absent in adult animals. This is surprising given that EC is an appealing mechanism for LC/NE neurons to synchronize together to cope with the global demand of NE signaling. However, our multi-cell patching recordings provide a direct demonstration of ECs established by mature LC/NE neurons with a comparable coupling rate, indicating that EC is abundant and still an important mechanism for LC/NE neurons to interact with each other in adult animals. A coupling rate ranging from $15 \%$ to $80 \%$ has been reported in 
many brain regions with the physiological recordings, based on pairwise testing of narrowly spaced cell pairs of the same cell type from juvenile animals ${ }^{37,49-51}$. While many coupling rates were reported without the information of the intersoma-distance (ISD), several studies indicate the coupling rate often drops to zero once the ISD is more than $40 \mu \mathrm{m}^{37,51-55}$. EC among mature LC neurons is generally consistent with the spatial and cell type-specific rule reported in other brain regions. While the overall coupling rate is low in our dataset, the coupling rate is much higher between cell pairs with ISD $<40$ $\mu \mathrm{m}$, particularly for FFs. Interestingly, when a pair of cells is more than $40 \mu \mathrm{m}$ apart, the possibility of finding an electrical connection, despite being very low, is much less distance-dependent, possibly due to the dendrodendritic form of electrical synapse formed in these neurons (see below). In addition, electrical coupling appears to decrease with age in most brain structures ${ }^{56}$, including in LC $^{20-22}$, but direct comparison to juvenile animals in this study indicates there is no significant difference in the coupling rate between juvenile and adult animals, indicating that EC in the LC may be less subject to developmental change than previously thought.

Gap junctions (GJs) generally form between cell pairs of the same type (homotypic GJ), while GJs between cell pairs of different cell types are rare or absent (heterotypic GJ) in most brain regions ${ }^{56}$. When we parsed out the coupling rate by the morphological cell type as we defined, we found that the rate was significantly affected by cell types: most ECs occur between cell pairs of the same morphological cell type, while EC is rare between cell pairs of the different cell type. This is still true when the ISD is controlled. Based on the spatial profiles of each type of electrical connection, neurons appear to form GJ exclusively with narrowly spaced, neighboring neurons of the same type (homotypic connection), while the heterotypic electrical connections typically form between cell pairs that are more spatially apart, despite being very rare. The result suggests that each LC neuron is intricately connected with their very close neighboring neurons of the same type to constitute cell type-specific subnetworks (FF subnetwork vs MP subnetwork), each of which may synchronize independently via extensive gap junctions. Of note, the coupling rate between FF-FF pairs is more than threefold higher than that between MP-MP pairs, suggesting the GJ-coupled MP subnetwork may be smaller in terms of cell population than the FF subnetwork. Alternatively, a lower coupling rate among MPs may suggest 
MPs are more heterogeneous than FFs. For instance, MPs in the core part of the LC are smaller in soma size than MPs in the ventral part of LC and they may be different in terms of projection targets and EC connectivity ${ }^{41,42}$. Given that FFs and MPs may have the differing preferential location and distinct projection targets, within-cell type electrical coupling is likely to promote the spread of electrical and chemical signals and spike synchronization within each subnetwork, exerting their synergetic effect on specific target regions.

While close appositions between two neurons do not necessarily mean electrical synapses, such contact sites identified under the light microscope could be reliably confirmed as real electrical synapses by follow-up electron microscope studies ${ }^{51,57-59}$. Our examination of close appositions between GJ-coupled pairs revealed more than $50 \%$ of GJ-coupled pairs have only one putative synapse, and multiple contact sites between pairs are less frequent. Almost all putative synapses were found to occur between dendrites, corroborating a previous LC study in rats ${ }^{24}$. In fact, dendrodendritic synapses appear to be a dominant form of electrical synapses across the brain regions $38,42,57,58,60,61$, while somatodendritic or somatosomatic ones are much less common 37,51 . In addition, electrical synapses in dendrites become further away from the soma when animals get older ${ }^{57,58}$. Consistent with these findings, putative synapses in mature LC were located most frequently in the middle of the entire dendritic trees of the GJ-coupled cell pair, with a similar dendritic distance away from each soma. Given electrical synapses located at such distal sites, fast signals like action potentials in prejunctional cells could be low-pass filtered and highly attenuated when propagating to postjunctional cells, giving rise to small-amplitude coupled potentials (like spikelet) at the somata of post-junctional neurons. How could such a small coupled potential drive synchronized firing and network oscillations under natural stimulation conditions? Recent work has shown those GJs found at distal dendritic sites can interact locally with chemical synaptic inputs to drive the synchronized spiking. While coupled potentials are small at the soma, these electrical signals are expected to be larger near the GJs, where they enable excitatory synaptic charge to spread into the dendrites of neighboring coupled neurons ${ }^{59}$, and/or sum with chemical synaptic inputs to generate dendritic spikes that actively propagate with little delay to the soma and drive action potentials with high fidelity. The former may promote the synchronized response 
of a GJ-coupled neuronal population to certain specific synaptic inputs, while the latter allows weak distal gap junction inputs to synchronize somatic action potentials, promoting synchronized spiking of GJ-coupled neurons upon common chemical synaptic input ${ }^{62-64}$. It will be interesting to investigate in future studies the mechanism(s) that LC neurons deploy to leverage their GJs to promote interneuronal synchrony.

\section{The network of GJ-coupled LC/NE neurons}

Gap junction is not just about connections between two neurons, instead it connects a group of neurons into a GJ-coupled network that coordinates/synergizes their activity as a whole. Thus, two questions ensue: what is the population size of each network, and how does an individual neuron in each network connect to other neurons of the same network? Despite extensive studies of gap junctions in many brain regions, these questions remain largely unexplored due to technical limitations. Gap junction studies with the dye-coupling approach may provide an insight into these questions ${ }^{37,50}$, but the neuron clusters labeled by this approach could result from the second-order coupling (in addition to first-order coupling) - these couplings are not otherwise discriminable - greatly compounding interpretation. In addition, the relative impermeability of different dyes to different kinds of electrical synapses results in many inconsistent results across studies and brain regions. In this regard, our multi-patch recordings confer some technical advantages over the dye-based approach. First, given GJs located at the dendrite far away from the soma, somatic membrane potentials in a pre-junctional cell are likely to be highly attenuated by the time they reach a post-junctional cell, since the signals need to first propagate into distal dendrites of pre-junctional cells, then traverse high-resistance gap junctions, and finally travel along the dendrites of post-junctional cells before propagating into the soma of post-junctional cells. It is reasonable to conclude that such highly attenuated coupled potential resulting from the first-order coupling can barely propagate any further into cells through second-order coupling. Thus, the GJ identified with pair-wise recordings should be exclusively first-order GJ, as interpreted by most if not all previous pair recordings, but this assertion has never been directly confirmed as yet. Our multi-cell recording sets allowed for directly testing said assertion and ruled out the possibility that GJ identified with pair-wise patch recordings could result from the second-order coupling. However, caution should 
be taken in interpreting the results if electrical synapses are located in the soma ${ }^{37}$, given that the signals passing through somatosomatic electrical synapses may be less attenuated. Given that electrical couplings we identified are confirmed as first-order, we can then examine the real organization of electrical connections beyond two cells in the context of the network. Second, our multiple-cell recording allows for identifying multiple GJ-coupled pairs across a cohort of up to eight cells, providing the first glimpse into how each neuron interacts with the rest of the neurons in a GJcoupled neuronal network.

With the support from the statistic simulation with a random connectivity model, a train-like pattern of two connections $(A \leftrightarrow B \leftrightarrow C)$ is overrepresented in our dataset. One might argue that said overrepresentation may be accounted by spatial and cell-type specific connectivity rules for electrical connections between LC/NE neurons that were empirically determined by us here. However, revising our model by adding these connectivity rules did not significantly improve the simulation results, and a train-like pattern of two connections $(A \leftrightarrow B \leftrightarrow C)$ remains unexplained even by models that incorporated such connectivity rules. All these simulations suggest that, in addition to spatial and cell type-specific rules in governing LC electrical connections, there is an additional train-like connectivity rule that may contribute to the overrepresentation of a train-like pattern of two connections in our dataset.

Furthermore, while the number for three connections is low, a train-like pattern ( $A \leftrightarrow B \leftrightarrow C \leftrightarrow D)$ is still a dominant pattern of three connections in our individual recording sets. Our simulation on the pattern of three connections yielded similar results and supported the idea of overrepresentation of a train-like connectivity pattern in LC/NE neurons. In addition, the overrepresentation can not be explained by the spatial and cell type-specific connectivity rule, further suggesting an additional train-like connectivity rule that governs the organization of three connections. While we could not test if this additional connectivity rule may apply to four or more connections for now (no four connections found in our dataset), the train-like connectivity rule may be a general wiring principle even applying to a whole GJcoupled network composed of hundreds of neurons (Fig.7C). Indeed, previous dye-coupled studies suggest this may be true in many brain regions and cell types, including $L C^{21,37,50,65}$. If a train-like organizational pattern is true, then the dye-injection of a single cell can only label the maximum of two 
additional cells if only first-order coupled cells are labeled. Indeed, the cluster of labeled cells in these studies are often small with a maximum of two additional labeled cells ${ }^{21,37}$. Some studies do label more than two additional cells, but those additional cells may be labeled via second-order coupling with a long dye injection time ${ }^{49,50}$. The most convincing data come from the mesencephalic trigeminal nucleus, where gap junctions between five neurons have been elegantly shown to arrange like a train-like pattern across neurons with immunostaining signals (See their figure $2 \mathrm{~L})^{37}$. However, the interpretation of these data is restrained by several issues inherent to this approach (see above), and whether or not this train-like connection pattern applies to the whole LC GJ-coupled network needs to be further explored in the LC, as well as in many other brain regions. In addition, the other two possible organization patterns for electrical connections, circular (Fig. 6D, right panel $4^{\text {th }}$ cartoon) or hub-like connection (see Fig. 6D, right panel $3^{\text {rd }}$ cartoon) patterns, were not observed in our dataset, suggesting that GJ-coupled pairs in LC may not be organized in these two patterns. However, simulated results on current data could not rule out the possibility that electrical connections in LC may be organized like circular and hub-like patterns. Model 3 simulations predicted 2.45 hub-like connections on average (95\% Cl: [0,5]) and predicted 1.39 circular-like connections on average $(95 \% \mathrm{Cl}:[0,4])$. While both average predicted numbers are numerically higher than the observed numbers of each (0), the observed numbers are still within statistical bounds. Recordings with more advanced techniques allowing for more than 8 simultaneous LC neuron recordings may be able to answer these questions in the future.

\section{Summary}

By profiling the morpho-electric heterogeneity and connectivity of a large number of mature noradrenergic neurons in the LC, we defined two major cell types in adult LC, and found that LC/NE neurons are wired via gap junctions into at least two cell type-specific GJ-coupled subnetworks. The assembly of these LC subnetworks follows a spatial and cell type-specific wiring principle that may be imposed by a unique train-like rule.

\section{Acknowledgments}


We thank AM's thesis committee members for their suggestions and comments, as well as the comments and support from the members in Jiang Lab. We acknowledge funding from NINDS R01

NS101596 (XJ, AM, MH), and NIMH R01 MH109556 (JJ, XJ, as well as Training Grant T32 EY07001

(AM) to support this work. Research reported in this publication was also supported by the Eunice Kennedy Shriver National Institute of Child Health \& Human Development of the National Institutes of Health under Award Number P50HD103555 for use of the Microscopy Core facilities. The content is solely the responsibility of the authors and does not necessarily represent the official views of the National Institutes of Health.

\section{Author contributions}

A. McKinney, X. Jiang designed the experiments. A. McKinney, X. Jiang, J. Jing performed the experiments. X. Jiang, A. McKinney, M. Hu, S. Patel, BR. Sheth, A. Hoskins, A. Mohammadyar, N. Naeem analyzed the data. X. Jiang wrote the manuscript and supervised all aspects of the project.

\section{Disclosure of Conflicts of Interest}

Authors declare no conflicts of interest.

\section{Materials and methods}

\section{Animals}

All experiments were performed according to the guidelines of the Institutional Animal Care and Use Committee (IACUC) of Baylor College of Medicine. Experiments on adult male and female mice (median age: 79, full range: 55--175 days) were performed using DBH-Cre/Ai9 ( $n=121)$ or wild-type ( $n=15)$. Crossing DBH (dopamine beta-hydroxylase)-Cre (Jackson Laboratory Stock No: 033951) mice, whose Cre recombinase expression is under the control of the promotor of DBH (a rate-limiting enzyme for norepinephrine synthesis), with Ai9 reporter mice globally labels all noradrenergic neurons, including LC neurons with the fluorescence marker tdTomato ${ }^{66}$. Additional younger DBH-Cre/Ai9 mice (P13-21, 
$\mathrm{n}=13$ ) were used to study connectivity between LC neurons at the juvenile age for comparison with the adult age.

\section{Slice preparation}

Slice preparation from adult mouse brainstem follows an N-Methyl-D-glucamine (NMDG) slicing protocol $^{26,27}$. Briefly, animals were deeply anesthetized using $3 \%$ isoflurane. After decapitation, the brain was removed and placed into cold $\left(0-4{ }^{\circ} \mathrm{C}\right)$ oxygenated NMDG solution containing $93 \mathrm{mM}$ NMDG, 93 mM HCl, 2.5 mM KCl, $1.2 \mathrm{mM} \mathrm{NaH}_{2} \mathrm{PO}_{4}, 30 \mathrm{mM} \mathrm{NaHCO}_{3}, 20$ mM HEPES, 25 mM glucose, $5 \mathrm{mM}$ sodium ascorbate, $2 \mathrm{mM}$ Thiourea, $3 \mathrm{mM}$ sodium pyruvate, $10 \mathrm{mM} \mathrm{MgSO}_{4}$ and $0.5 \mathrm{mM} \mathrm{CaCl}_{2}, \mathrm{pH}$ 7.35 (all from SIGMA-ALDRICH). Horizontal slices were prepared using a vibratome (200 $\mu \mathrm{m}$ thick) using zirconia ceramic blades. The brain slices were kept at $37.0 \pm 0.5^{\circ} \mathrm{C}$ in oxygenated NMDG solution for 10 minutes, and then were transferred to an artificial cerebrospinal fluid (ACSF) containing $125 \mathrm{mM} \mathrm{NaCl}, 2.5 \mathrm{mM} \mathrm{KCl} 1.25 \mathrm{mM} \mathrm{NaH}_{2} \mathrm{PO}_{4}, 25 \mathrm{mM} \mathrm{NaHCO}_{3}, 1 \mathrm{mM} \mathrm{MgCl}, 25 \mathrm{mM}$ glucose, and 2 $\mathrm{mM} \mathrm{CaCl}_{2}(\mathrm{pH} 7.4)$ for at least 1 hour prior to the beginning of recordings. During the recording sessions, the slices were submerged in a custom chamber and were stabilized with a fine nylon net attached to a custom-designed platinum ring. This recording chamber was continuously perfused with oxygenated ACSF throughout the recording session. Brainstem slices from juvenile mice were prepared following a similar protocol except for the cutting solution (with regular ACSF, not NMDG). For the recording of hippocampal neurons, the parasagittal cortical slices were prepared following the same protocol for adult animals.

\section{Electrophysiology}

Whole-cell recordings were performed as described previously ${ }^{27,31,32}$. Briefly, patch pipettes (4-7 M 2 ) were filled with an internal solution containing $120 \mathrm{mM}$ potassium gluconate, $10 \mathrm{mM}$ HEPES, $4 \mathrm{mM}$ $\mathrm{KCl}, 4 \mathrm{mM} \mathrm{MgATP}, 0.3 \mathrm{mM} \mathrm{Na}{ }_{3} \mathrm{GTP}, 10 \mathrm{mM}$ sodium phosphocreatine and $0.5 \%$ biocytin (pH 7.25). Simultaneous whole-cell recordings from up to 8 neurons were performed using two Quadro EPC 10 amplifiers (HEKA Electronic, Germany). PatchMaster (HEKA) and custom-written Matlab-based programs (Mathworks) were used to operate the recording system and perform online and offline data analysis. In current-clamp recordings, neurons were first current clamped at $\sim-40 p A$ to prevent its 
spontaneous firings (see results). Action potentials were evoked by current injection into presynaptic neurons at $2 \mathrm{nA}$ for $2 \mathrm{~ms}$ at $0.05 \mathrm{~Hz}$ for 20-50 trials, and the average of the sweeps in postsynaptic cells was used to detect synaptic connections. The spike threshold and firing pattern in response to sustained depolarizing currents were recorded for each neuron by injecting increasing current steps $(+10 \mathrm{pA})$.

For coupling coefficient (CC) calculations in gap junctions, hyperpolarizing current pulses of 600 ms duration were injected into one cell and resulting voltage deflections were measured in both cells. The voltage deflections were measured at the last 10-20 ms of current injections given the large time constant of the membrane potential responses of two cells (see Results). The coupling coefficient from cell 1 to cell 2 (CC1) was defined as V2/V1 and the coupling coefficient in the opposite direction from cell 2 to cell 1 (CC2) was defined as V1/ V2, where V1 is the voltage deflection in one cell and V2 is the corresponding voltage deflection in the other cell. To improve the signal-to-noise ratio, a total of 10 to 30 single responses were often generated and averaged. For each of the coupled pairs, the mean CC was calculated as the average from the values in both directions.

\section{Electrophysiological features extraction}

A custom-made MATLAB software kit was developed to analyze the electrophysiology data offline. We extracted 13 electrophysiological properties from each neuron, following a similar method as used in previous studies ${ }^{48,49}$. The rheobase refers to the minimal stimulation current to elicit any action potentials. To extract the properties of action potential (AP), we used the first AP elicited by our step stimulation protocol. To calculate the threshold of the AP, the trace within 20 ms preceding the AP peak was used to calculate the third derivative first and the data point with the maximal third derivative value was defined as the threshold of the AP. The AP amplitude is the difference between the threshold potential and the peak potential of the AP. The AP width was defined as that at half-height. The time range from the threshold point to peak is 'rising phase time' while 'decay phase time' is the time range from the peak to the potential equal to the threshold. The afterhyperpolarization was defined as the voltage difference between the threshold and the minimal value of the trough after the AP. The time range from the stimulation onset to the threshold of the first AP at the rheobase sweep was defined as the AP delay 
time.

To calculate the time constant $\tau$, we fitted each hyperpolarizing trace (from the stimulation onset to the minimal potential within the first-half stimulation period) with an exponential function as follows:

$$
f(t)=a e^{(-t / \tau)}+C
$$

Here, $t$ is the time relative to the stimulation onset, $a$ and $C$ are two constants, $f(t)$ is the current value at time $t$, and $\tau$ is the membrane constant. Then the $\tau$ was calculated for each trace and averaged across all traces.

To calculate the maximal firing rate, the depolarizing trace with the maximal number of APs elicited by the step protocol was used to count the number of APs, which was then divided by the stimulation duration (600 ms). To calculate the adaptive index, the inter-spike intervals (ISIs) were first measured, and then the ratio of the last and the first ISIs was calculated. The CV was calculated as the ratio of the standard deviation of ISIs and the mean of ISIs. To compare the first AP and the subsequent ones, we calculated the ratio of their AP amplitude and the ratio of their half-widths. The amplitude and half-width of the first AP were first calculated and then divided by the mean of these values of the subsequent APs.

To calculate the input resistance, we measured the steady-state membrane potentials in the traces elicited by the first five negative currents in the step protocol, and then plotted these values ( $y$ axis) against each of the corresponding currents (x-axis). We fitted the plot with a linear function and the slope of the fitting line is the input resistance, and the potential at the x-axis intersection is the RMP.

\section{Morphological reconstructions and analysis}

Morphological reconstructions and analysis were performed using a 100X oil immersion objective lens and camera lucida system using Neurolucida (MicroBrightField), following the same procedure as previously described ${ }^{27,31,32}$. Briefly, neurons were filled with biocytin during the recording, and slices were fixed right after recordings by immersion in freshly prepared $2.5 \%$ glutaraldehyde (from Electron Microscopy Science Cat.no. 16220), 4\% paraformaldehyde (from SIGMA-ALDRICH Cat.no. P6148) in 0.1 M phosphate-buffered saline at $4^{\circ} \mathrm{C}$ for $7-10$ days, and then processed with the avidin-biotin- 
peroxidase method to reveal cell morphology. Slices were then mounted in an aqueous Mowiol mounting solution (SIGMA-ALDRICH) and the morphologically recovered cells were examined, reconstructed, and analyzed using a 100X oil-immersion objective lens and camera lucida system. Tissue shrinkage due to the fixation procedure was not compensated. The shrinkage of the tissue surrounding the biocytin-stained cells is about $10-20 \%$, consistent with previous studies ${ }^{31,67}$. The reconstructions were not corrected for tissue shrinkage.

Dendritic arbor and axonal structure of reconstructed morphologies of $n=70$ cells were analyzed using Neurolucida software. Additional morphological analysis was performed using custom Python code.

\section{Data Analysis}

Data analysis was performed using custom-written MATLAB software and Python routines. For statistical comparisons, the normality of the data was first tested using the Shapiro-Wilk test. If compared groups show normal distributions, Student's t-test or the paired t-test was used; otherwise, the non-parametric paired Wilcoxon-sign rank test and Mann-Whitney test were used. The paired t-test and the non-parametric paired Wilcoxon-sign rank test were used for comparison of data collected before and after drug application from an individual neuron; Student's t-test and Mann-Whitney test were used for two independent groups. One way or two-way ANOVA was also used for comparing morpho-electric features of different types of neurons.

\section{Data Simulation}

\section{Model 1: random connectivity model}

First, the overall pairwise connection probability $(P)$ was obtained from the recordings and is the ratio of the observed number of electrical connections to the total number of potential electrical connections tested across all recordings, regardless of the neuronal type and inter-neuronal distance. Expected numbers of double or 2-connections were calculated based on pairwise connection probabilities. For instance, if there were four neurons $A, B, C$, and D in a particular recording/slice, then the expected value of a non-train-like 2-connection $A B-C D$ was calculated to be equal to $P$ (pairwise connection) * $\mathrm{P}$ (pairwise connection). The expected number of train-like and non-train-like 2-connections for a given 
slice was the sum of the expected values of all 2-connections for the recording. The grand total expected number of 2-connections of a given type was the sum of the corresponding expected number values over all our recordings. Similar simulations were conducted for triple or 3-connections, and the product of three pairwise connections was used for all calculations.

\section{Model 2: Differentiated neuronal types}

For each individual recording (slice) for which the slice was fixed and neuronal types (FF - fusiform cell type; MP - multipolar cell type) were identified, we calculated expected numbers of train-like and nontrain-like 2- and 3-connections in the following way. For a given pair of neurons from a given recording slice, its corresponding pairwise connection probability (FF-FF, FF-MP, or MP-MP depending on the morphological cell types of the neurons constituting the pair) across all recordings, which was computed earlier, was used for all calculations (Fig.4G). Expected numbers of 2-connections were calculated based on pairwise connection probabilities. For instance, if neurons $A, B, C$, and $D$ in a particular recording/slice were FF, FF, MP, and MP neuronal types respectively, then the expected value of a train-like 2-connection $A B-B C$ was calculated to be equal to $P(F F-F F$ connection) * $P(F F-M P$ connection). Similar calculations were performed for all possible 2-connections (e.g. train-like 2connection $A B-C D$ and non-train-like connections $A B-C D$ in the above example). The expected number of train-like and non-train-like 2-connections for a given slice was then the sum of the individual probabilities thus calculated. The grand total expected number of 2-connections of a given type was computed by summing the corresponding expected number values over all our recordings. Calculations to compute the expected values of triple or 3-connections were similar, with the exception that the expected value was now a product of three, and not two, pairwise connections; in the above example, $\mathrm{P}(\mathrm{AB}-\mathrm{BC}-\mathrm{CD})=\mathrm{P}($ FF-FF connection $){ }^{*} \mathrm{P}(\mathrm{FF}-\mathrm{MP}$ connection $){ }^{*} \mathrm{P}(\mathrm{MP}-\mathrm{MP}$ connection $)$.

\section{Model 3: Differentiated neuronal types, short-range connectivity}

In addition to the constraints specified in Model 2, namely pairwise connection probabilities based on neuronal identity, the following additional constraints were added into Model 3. First, because connections between neuronal pairs were largely non-existent for inter-neuronal distances $>80 \mu \mathrm{m}$, all neuronal pairs in the model that were $>80 \mu \mathrm{m}$ apart were taken to have no connection. Second, 
because neurons of the same type clustered together in the recordings, FF-FF and MP-MP connection probabilities for short distances $(16-40 \mu \mathrm{m})$ were used, and because neurons of different types were further apart in the recordings, FF-MP connection probability for 0-80 $\mathrm{mm}$ distance was used in the model. The remaining computations were performed in accord with those of the previous model.

\section{Monte-Carlo simulations}

On each of 1000 runs of the simulation, we counted the number of 2- and 3-connections from all simulated recordings. Each simulated recording matched one-to-one a biological recording in terms of number and type of neurons. A random number generated by the random number generator determined whether a connection existed between each neuron in the corresponding simulated recording. If the random number was lower than the expected value of the connection (different values based on whether the corresponding connection in the recording was FF-FF/FF-MP/MP-MP), the simulated connection was considered to exist. For each run, simulated double and triple connections were obtained for each recording and the total simulated number was obtained by summing over all the simulated recordings. Summary statistics were obtained from the simulated data from 1000 such runs: i) the mean, standard deviation, and $95 \%$ confidence intervals for the simulated numbers of train-like and non-train-like 2- and 3-connections, and ii) the probability that the ratio of the simulated train-like over non-train-like 2-connections (3-connections) was greater than the ratio of the observed number of train-like over non-train-like 2-connections (3-connections). All simulations were conducted on MATLAB (R2021a, The Mathworks, Natick, MA).

\section{References}

1 Schwarz, L. A. \& Luo, L. Organization of the locus coeruleus-norepinephrine system. Curr Bio/ 25, R1051R1056, doi:10.1016/j.cub.2015.09.039 (2015).

2 Bast, N., Poustka, L. \& Freitag, C. M. The locus coeruleus-norepinephrine system as pacemaker of attention - a developmental mechanism of derailed attentional function in autism spectrum disorder. Eur J Neurosci 47, 115-125, doi:10.1111/ejn.13795 (2018). 
3 Southwick, S. M. et al. Role of norepinephrine in the pathophysiology and treatment of posttraumatic stress disorder. Biol Psychiatry 46, 1192-1204, doi:10.1016/s0006-3223(99)00219-x (1999).

4 Weinshenker, D. Long Road to Ruin: Noradrenergic Dysfunction in Neurodegenerative Disease. Trends Neurosci 41, 211-223, doi:10.1016/j.tins.2018.01.010 (2018).

5 Fortress, A. M. et al. Designer receptors enhance memory in a mouse model of Down syndrome. $J$ Neurosci 35, 1343-1353, doi:10.1523/JNEUROSCI.2658-14.2015 (2015).

6 Sara, S. J. \& Bouret, S. Orienting and reorienting: the locus coeruleus mediates cognition through arousal. Neuron 76, 130-141, doi:10.1016/j.neuron.2012.09.011 (2012).

7 Bari, A. et al. Differential attentional control mechanisms by two distinct noradrenergic coeruleo-frontal cortical pathways. Proc Natl Acad Sci U S A 117, 29080-29089, doi:10.1073/pnas.2015635117 (2020).

8 Hirschberg, S., Li, Y., Randall, A., Kremer, E. J. \& Pickering, A. E. Functional dichotomy in spinal- vs prefrontal-projecting locus coeruleus modules splits descending noradrenergic analgesia from ascending aversion and anxiety in rats. Elife 6, doi:10.7554/eLife.29808 (2017).

$9 \mathrm{Li}, \mathrm{Y}$. et al. Retrograde optogenetic characterization of the pontospinal module of the locus coeruleus with a canine adenoviral vector. Brain Res 1641, 274-290, doi:10.1016/j.brainres.2016.02.023 (2016). Chandler, D. J., Gao, W.-J. \& Waterhouse, B. D. Heterogeneous organization of the locus coeruleus projections to prefrontal and motor cortices. Proceedings of the National Academy of Sciences 111, 6816-6821, doi:10.1073/pnas.1320827111 (2014).

11 Totah, N. K. B., Logothetis, N. K. \& Eschenko, O. Noradrenergic ensemble-based modulation of cognition over multiple timescales. Brain Res 1709, 50-66, doi:10.1016/j.brainres.2018.12.031 (2019).

12 Uematsu, A. et al. Modular organization of the brainstem noradrenaline system coordinates opposing learning states. Nat Neurosci 20, 1602-1611, doi:10.1038/nn.4642 (2017).

13 Liu, R. H., Fung, S. J., Reddy, V. K. \& Barnes, C. D. Localization of glutamatergic neurons in the dorsolateral pontine tegmentum projecting to the spinal cord of the cat with a proposed role of glutamate on lumbar motoneuron activity. Neuroscience 64, 193-208, doi:https://doi.org/10.1016/03064522(94)00354-8 (1995).

14 Fung, S. I. et al. Cotransmitter-mediated locus coeruleus action on motoneurons. Brain Res Bull 35, 423432, doi:10.1016/0361-9230(94)90155-4 (1994).

15 Yang, B. et al. Locus coeruleus anchors a trisynaptic circuit controlling fear-induced suppression of feeding. Neuron, doi:10.1016/j.neuron.2020.12.023 (2021).

16 Aghajanian, G. K. \& VanderMaelen, C. P. alpha 2-adrenoceptor-mediated hyperpolarization of locus coeruleus neurons: intracellular studies in vivo. Science 215, 1394-1396, doi:10.1126/science.6278591 (1982).

17 Egan, T. M., Henderson, G., North, R. A. \& Williams, J. T. Noradrenaline-mediated synaptic inhibition in rat locus coeruleus neurones. J Physiol 345, 477-488, doi:10.1113/jphysiol.1983.sp014990 (1983). Williams, J. T., Henderson, G. \& North, R. A. Characterization of alpha 2-adrenoceptors which increase potassium conductance in rat locus coeruleus neurones. Neuroscience 14, 95-101, doi:10.1016/03064522(85)90166-6 (1985).

19 Huang, H. P. et al. Long latency of evoked quantal transmitter release from somata of locus coeruleus neurons in rat pontine slices. Proc Natl Acad Sci U S A 104, 1401-1406, doi:10.1073/pnas.0608897104 (2007).

20 Christie, M. J., Williams, J. T. \& North, R. A. Electrical coupling synchronizes subthreshold activity in locus coeruleus neurons in vitro from neonatal rats. J Neurosci 9, 3584-3589 (1989).

21 Christie, M. J. \& Jelinek, H. F. Dye-coupling among neurons of the rat locus coeruleus during postnatal development. Neuroscience 56, 129-137, doi:10.1016/0306-4522(93)90568-z (1993).

22 Travagli, R. A., Dunwiddie, T. V. \& Williams, J. T. Opioid inhibition in locus coeruleus. J Neurophysiol 74, 518-528 (1995).

23 Patrone, L. G., Bicego, K. C., Hartzler, L. K., Putnam, R. W. \& Gargaglioni, L. H. Cardiorespiratory effects of gap junction blockade in the locus coeruleus in unanesthetized adult rats. Respir Physiol Neurobiol 190, 86-95, doi:10.1016/j.resp.2013.09.001 (2014). 
24 Ishimatsu, M. \& Williams, J. T. Synchronous activity in locus coeruleus results from dendritic interactions in pericoerulear regions. J Neurosci 16, 5196-5204 (1996).

25 Rash, J. E. et al. Identification of connexin36 in gap junctions between neurons in rodent locus coeruleus. Neuroscience 147, 938-956, doi:10.1016/j.neuroscience.2007.04.061 (2007).

26 Ting, J. T., Daigle, T. L., Chen, Q. \& Feng, G. Acute brain slice methods for adult and aging animals: application of targeted patch clamp analysis and optogenetics. Methods Mol Biol 1183, 221-242, doi:10.1007/978-1-4939-1096-0_14 (2014).

27 Jiang, X. et al. Principles of connectivity among morphologically defined cell types in adult neocortex. Science 350, aac9462, doi:10.1126/science.aac9462 (2015).

28 Ballantyne, D., Andrzejewski, M., Muckenhoff, K. \& Scheid, P. Rhythms, synchrony and electrical coupling in the Locus coeruleus. Respir Physiol Neurobiol 143, 199-214, doi:10.1016/j.resp.2004.07.018 (2004).

29 Andrzejewski, M., Muckenhoff, K., Scheid, P. \& Ballantyne, D. Synchronized rhythms in chemosensitive neurons of the locus coeruleus in the absence of chemical synaptic transmission. Respir Physiol 129, 123-140, doi:10.1016/s0034-5687(01)00300-0 (2001).

30 Kuo, C. C. et al. Inhibitory interneurons regulate phasic activity of noradrenergic neurons in the mouse locus coeruleus and functional implications. J Physiol 598, 4003-4029, doi:10.1113/JP279557 (2020).

31 Scala, F. et al. Layer 4 of mouse neocortex differs in cell types and circuit organization between sensory areas. Nat Commun 10, 4174, doi:10.1038/s41467-019-12058-z (2019).

32 Scala, F. et al. Phenotypic variation of transcriptomic cell types in mouse motor cortex. Nature, doi:10.1038/s41586-020-2907-3 (2020).

33 Swanson, L. W. The locus coeruleus: a cytoarchitectonic, Golgi and immunohistochemical study in the albino rat. Brain Res 110, 39-56, doi:10.1016/0006-8993(76)90207-9 (1976).

34 Blythe, S. N., Wokosin, D., Atherton, J. F. \& Bevan, M. D. Cellular mechanisms underlying burst firing in substantia nigra dopamine neurons. J Neurosci 29, 15531-15541, doi:10.1523/JNEUROSCI.2961-09.2009 (2009).

35 Fung, S. J., Reddy, V. K., Liu, R. H., Wang, Z. \& Barnes, C. D. Existence of glutamate in noradrenergic locus coeruleus neurons of rodents. Brain Res Bull 35, 505-512, doi:10.1016/0361-9230(94)90164-3 (1994).

36 Zhu, J. J., Esteban, J. A., Hayashi, Y. \& Malinow, R. Postnatal synaptic potentiation: delivery of GluR4containing AMPA receptors by spontaneous activity. Nat Neurosci 3, 1098-1106, doi:10.1038/80614 (2000).

37 Curti, S., Hoge, G., Nagy, J. I. \& Pereda, A. E. Synergy between electrical coupling and membrane properties promotes strong synchronization of neurons of the mesencephalic trigeminal nucleus. $J$ Neurosci 32, 4341-4359, doi:10.1523/JNEUROSCI.6216-11.2012 (2012).

38 Hidaka, S., Akahori, Y. \& Kurosawa, Y. Dendrodendritic electrical synapses between mammalian retinal ganglion cells. J Neurosci 24, 10553-10567, doi:10.1523/JNEUROSCI.3319-04.2004 (2004).

39 Bennett, M. V. \& Zukin, R. S. Electrical coupling and neuronal synchronization in the Mammalian brain. Neuron 41, 495-511, doi:10.1016/s0896-6273(04)00043-1 (2004).

40 Erdos, P. \& Rényi, A. On the evolution of random graphs. Transactions of the American Mathematical Society 286, 257-257 (1984).

41 Loughlin, S. E., Foote, S. L. \& Grzanna, R. Efferent projections of nucleus locus coeruleus: morphologic subpopulations have different efferent targets. Neuroscience 18, 307-319, doi:10.1016/03064522(86)90156-9 (1986).

42 Waterhouse, B. D., Lin, C. S., Burne, R. A. \& Woodward, D. J. The distribution of neocortical projection neurons in the locus coeruleus. J Comp Neurol 217, 418-431, doi:10.1002/cne.902170406 (1983).

43 Totah, N. K., Neves, R. M., Panzeri, S., Logothetis, N. K. \& Eschenko, O. The Locus Coeruleus Is a Complex and Differentiated Neuromodulatory System. Neuron 99, 1055-1068 e1056, doi:10.1016/j.neuron.2018.07.037 (2018).

44 Zeisel, A. et al. Brain structure. Cell types in the mouse cortex and hippocampus revealed by single-cell RNA-seq. Science 347, 1138-1142, doi:10.1126/science.aaa1934 (2015). 
45 Tasic, B. et al. Adult mouse cortical cell taxonomy revealed by single cell transcriptomics. Nat Neurosci 19, 335-346, doi:10.1038/nn.4216 (2016).

46 Trudeau, L. E. Glutamate co-transmission as an emerging concept in monoamine neuron function. J Psychiatry Neurosci 29, 296-310 (2004).

47 Cedarbaum, J. M. \& Aghajanian, G. K. Noradrenergic neurons of the locus coeruleus: inhibition by epinephrine and activation by the alpha-antagonist piperoxane. Brain Res 112, 413-419, doi:10.1016/0006-8993(76)90297-3 (1976).

48 Ho"kfelt, T., Fuxe, K., Goldstein, M. \& Johansson, O. Immunohistochemical evidence for the existence of adrenaline neurons in the rat brain. Brain Research 66, 235-251 (1974).

49 Devor, A. \& Yarom, Y. Electrotonic coupling in the inferior olivary nucleus revealed by simultaneous double patch recordings. J Neurophysiol 87, 3048-3058, doi:10.1152/jn.2002.87.6.3048 (2002).

50 Long, M. A., Landisman, C. E. \& Connors, B. W. Small clusters of electrically coupled neurons generate synchronous rhythms in the thalamic reticular nucleus. J Neurosci 24, 341-349, doi:10.1523/JNEUROSCI.3358-03.2004 (2004).

51 Tamas, G., Buhl, E. H., Lorincz, A. \& Somogyi, P. Proximally targeted GABAergic synapses and gap junctions synchronize cortical interneurons. Nat Neurosci 3, 366-371, doi:10.1038/73936 (2000).

52 Beierlein, M., Gibson, J. R. \& Connors, B. W. A network of electrically coupled interneurons drives synchronized inhibition in neocortex. Nat Neurosci 3, 904-910, doi:10.1038/78809 (2000).

53 Gibson, J. R., Beierlein, M. \& Connors, B. W. Two networks of electrically coupled inhibitory neurons in neocortex. Nature 402, 75-79, doi:10.1038/47035 (1999).

54 Galarreta, M. \& Hestrin, S. Electrical and chemical synapses among parvalbumin fast-spiking GABAergic interneurons in adult mouse neocortex. Proc Natl Acad Sci U S A 99, 12438-12443, doi:10.1073/pnas.192159599 (2002).

55 Galarreta, M. \& Hestrin, S. Electrical synapses between GABA-releasing interneurons. Nat Rev Neurosci 2, 425-433, doi:10.1038/35077566 (2001).

56 Peinado, A., Yuste, R. \& Katz, L. C. Gap Junctional Communication and the Development of Local Circuits in Neocortex. Cerebral Cortex 3, 488-498, doi:10.1093/cercor/3.5.488 (1993).

57 Fukuda, T. \& Kosaka, T. Gap junctions linking the dendritic network of GABAergic interneurons in the hippocampus. J Neurosci 20, 1519-1528 (2000).

58 Fukuda, T. \& Kosaka, T. Ultrastructural study of gap junctions between dendrites of parvalbumincontaining GABAergic neurons in various neocortical areas of the adult rat. Neuroscience 120, 5-20, doi:10.1016/s0306-4522(03)00328-2 (2003).

59 Vervaeke, K., Lorincz, A., Nusser, Z. \& Silver, R. A. Gap junctions compensate for sublinear dendritic integration in an inhibitory network. Science 335, 1624-1628, doi:10.1126/science.1215101 (2012).

60 Sotelo, C., Llinas, R. \& Baker, R. Structural study of inferior olivary nucleus of the cat: morphological correlates of electrotonic coupling. J Neurophysio/ 37, 541-559, doi:10.1152/jn.1974.37.3.541 (1974).

61 Vervaeke, K. et al. Rapid desynchronization of an electrically coupled interneuron network with sparse excitatory synaptic input. Neuron 67, 435-451, doi:10.1016/j.neuron.2010.06.028 (2010).

62 Migliore, M., Hines, M. L. \& Shepherd, G. M. The role of distal dendritic gap junctions in synchronization of mitral cell axonal output. J Comput Neurosci 18, 151-161, doi:10.1007/s10827-005-6556-1 (2005).

63 Trenholm, S. et al. Nonlinear dendritic integration of electrical and chemical synaptic inputs drives finescale correlations. Nat Neurosci 17, 1759-1766, doi:10.1038/nn.3851 (2014).

64 Schoppa, N. E. \& Westbrook, G. L. AMPA autoreceptors drive correlated spiking in olfactory bulb glomeruli. Nat Neurosci 5, 1194-1202, doi:10.1038/nn953 (2002).

65 Lee, S. C., Patrick, S. L., Richardson, K. A. \& Connors, B. W. Two functionally distinct networks of gap junction-coupled inhibitory neurons in the thalamic reticular nucleus. J Neurosci 34, 13170-13182, doi:10.1523/JNEUROSCI.0562-14.2014 (2014).

66 Rukhadze, I. et al. Catecholaminergic A1/C1 neurons contribute to the maintenance of upper airway muscle tone but may not participate in NREM sleep-related depression of these muscles. Respir Physiol Neurobiol 244, 41-50, doi:10.1016/j.resp.2017.07.001 (2017). 
bioRxiv preprint doi: https://doi.org/10.1101/2022.03.01.481656; this version posted March 3, 2022. The copyright holder for this preprint (which was not certified by peer review) is the author/funder. All rights reserved. No reuse allowed without permission.

67 Markram, H., Lubke, J., Frotscher, M., Roth, A. \& Sakmann, B. Physiology and anatomy of synaptic connections between thick tufted pyramidal neurones in the developing rat neocortex. J Physio/ $\mathbf{5 0 0}$ ( Pt 2), 409-440, doi:10.1113/jphysiol.1997.sp022031 (1997). 


\section{Figure S1}

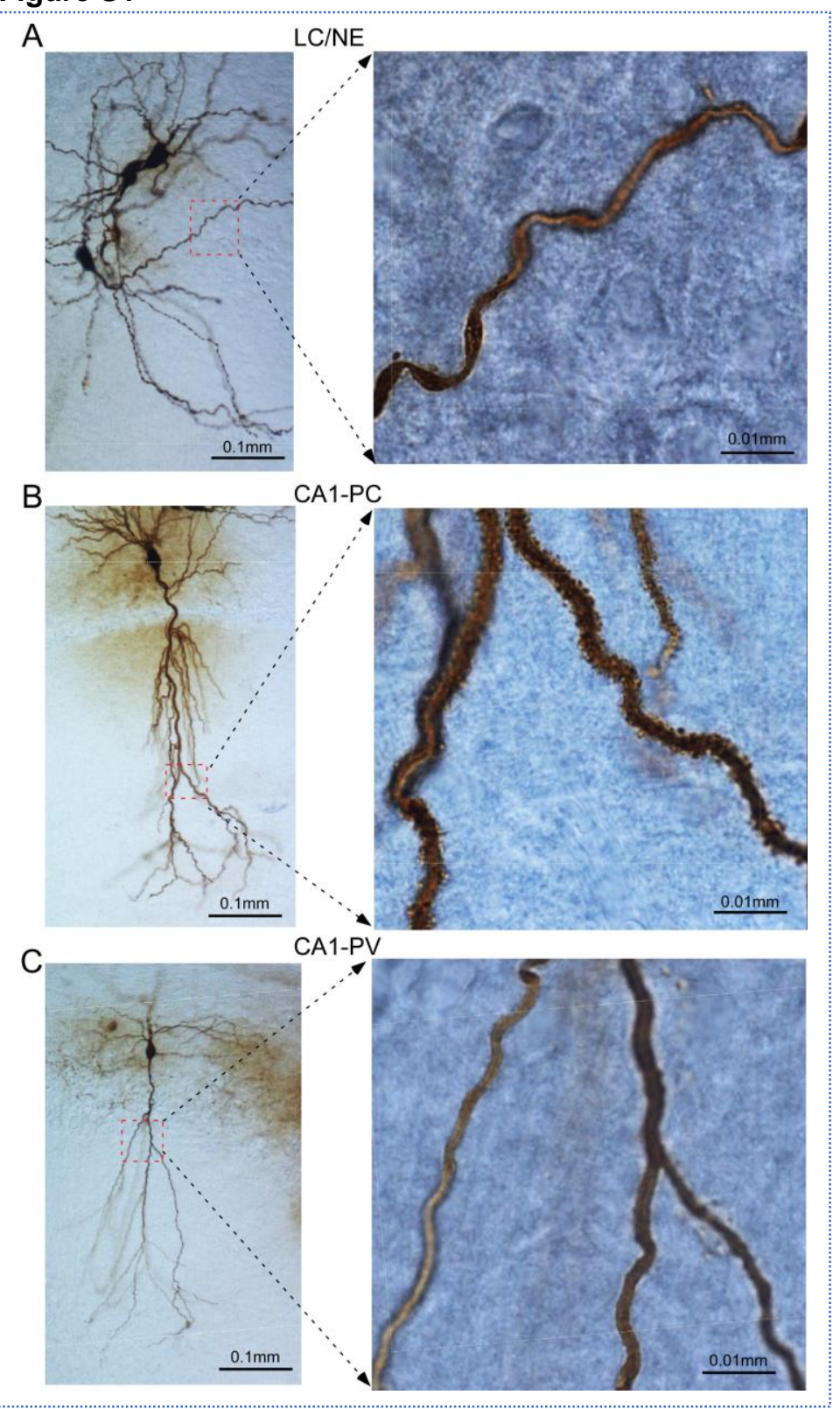

Figure S1. Dendrites of LC/NE neurons compared to CA1 hippocampal pyramidal neurons (CA1PCs) and PV-expressing interneuron (CA1-PV). (A) Left: A photomicrograph depicting the morphological recovery of three LC/NE neurons; Right: high-magnification view of a dendritic section (squared on the left with red dashline) of one of the same LC/NE neurons. (B) Left: A photomicrograph depicting the morphological recovery of a CA1-PC; Right: high-magnification view of dendritic sections (squared on the left with red dashline) of the same CA1-PC. (C) Left: A photomicrograph depicting the morphological recovery of a CA1-PV; Right: high-magnification view of dendritic sections (squared on the left with red dashline) of the same CA1-PV. 
Figure S2

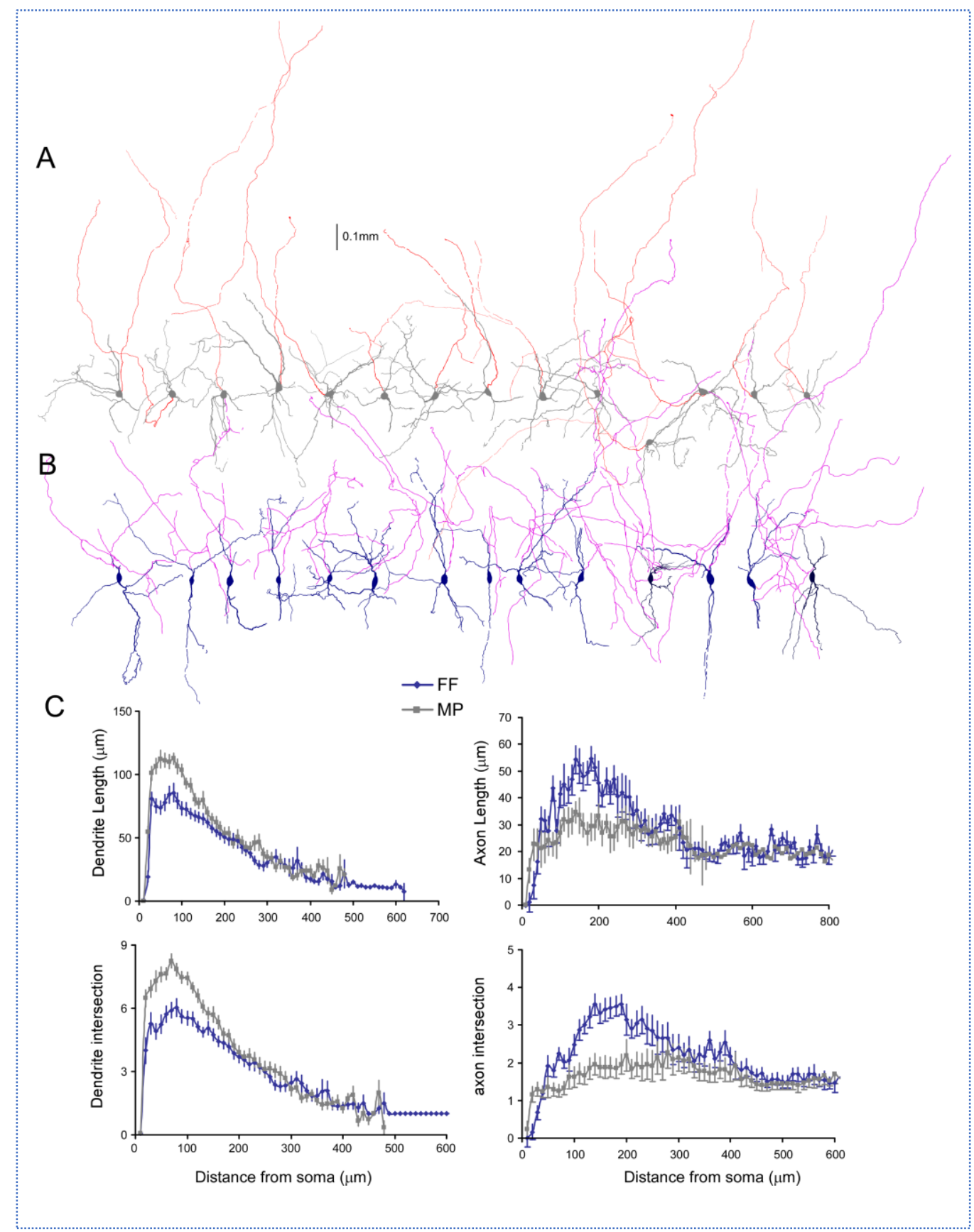

Figure S2. Comparison of axonal and dendritic arbors of two LC/NE morphological cell types. (A) Morphology of multipolar cells (MPs) including all axonal trees remained in slices. The dendrite and soma are shown in gray and the axon is shown in red. (B) Morphology of fusiform cells (FFs) including all axonal trees remained in slices. The dendrite and soma are shown in dark blue, and the axon is shown in purple. (C) Sholl analysis of length and intersections of both axonal and dendritic arbors of MP and FF. Top left: Dendrite length (FF vs MP: $F(1)=130.6 ; p<0.0001$ with two-way ANOVA, $n=32$ for $F F$ and $n=36$ for MP); Bottom left: dendrite intersection (FF vs MP: $F(1)=132.9 ; p<0.0001$ with two-way ANOVA, $n=32$ for $F F$ and $n=36$ for MP). Top Right: axonal length (FF vs MP: $F(1)=47.3 ; p<0.0001$ with two-way ANOVA, $n=25$ for $F F$ and $n=31$ for MP); Bottom right: axonal intersection (FF vs MP: $F(1)=87.3 ; p<0.0001$ with two-way ANOVA, $n=25$ for FF and $n=31$ for MP). 


\section{Figure S3}

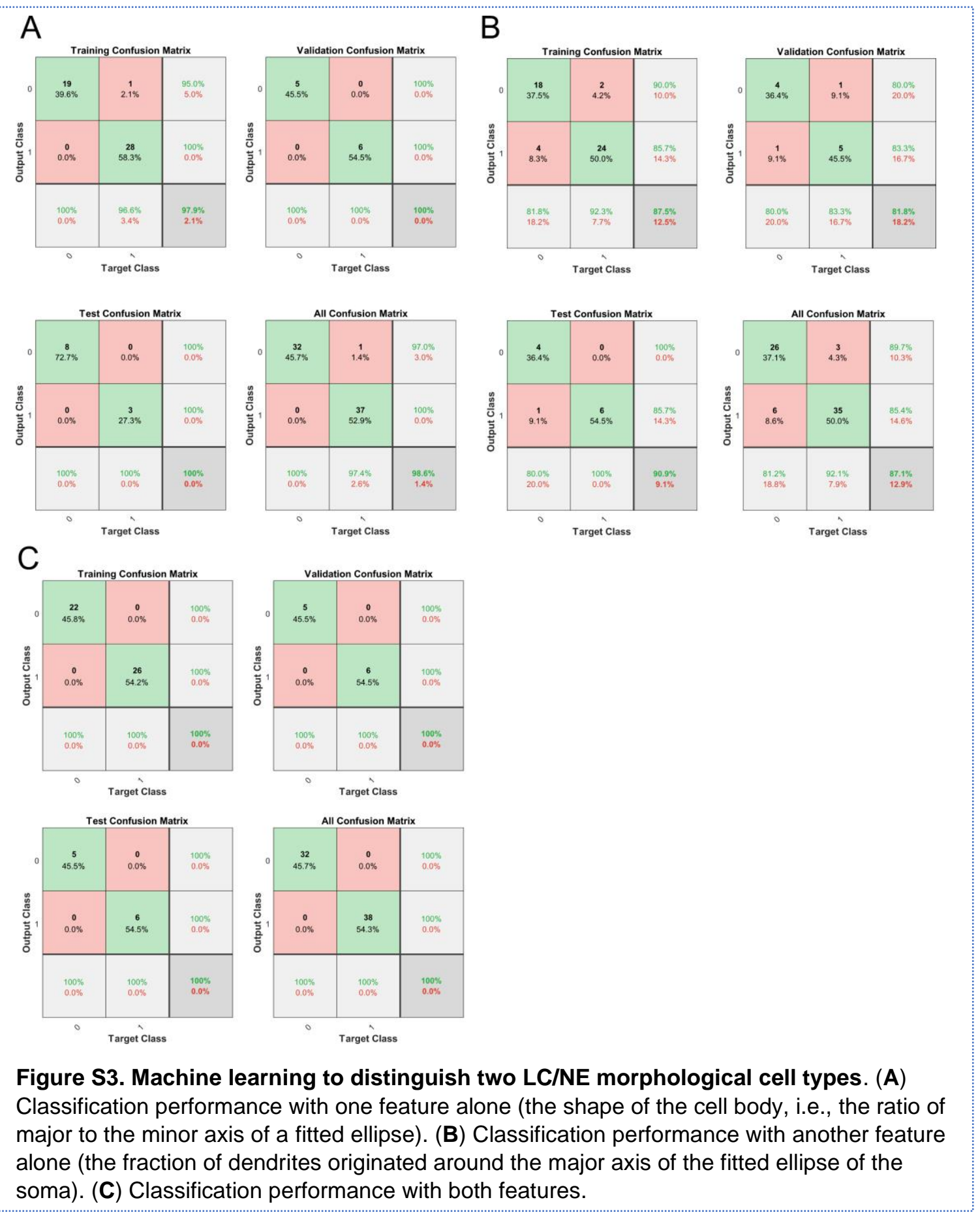


Figure $\mathbf{S 4}$

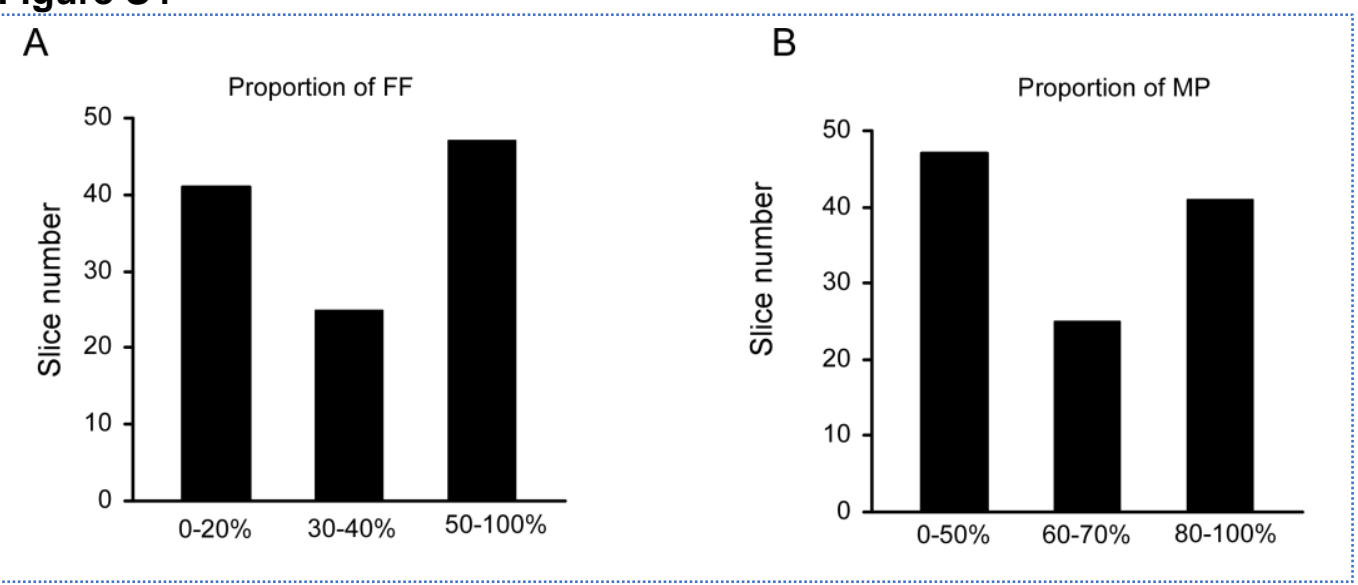

Figure S4. The proportion of FF or MP in individual recording sets (slice). (A) Histogram of the proportion of FF across all slices. (B) Histogram of the proportion of MPs across all slices. 
Supplemental Table 1: Electrophysiological Properties of Multipolar cells (MPs) and Fusiform cells (FFs)

\begin{tabular}{r|c|c|}
\hline Cell Type & MP (n=41) & FF (n=19) \\
\hline AP threshold $(\mathrm{mV})$ & $-31.16 \pm 0.91$ & $-30.93 \pm 1.53$ \\
AP amplitude $(\mathrm{mV})$ & $69.62 \pm 1.55$ & $77.38 \pm 1.55^{* *}$ \\
AP half width (ms) & $2.20 \pm 0.07$ & $1.84 \pm 0.06^{* *}$ \\
AP upstroke-to-downstroke ratio & $3.29 \pm 0.09$ & $3.68 \pm 0.17^{*}$ \\
Resting membrane potential (mV) & $-44.82 \pm 1.46$ & $-44.92 \pm 2.12$ \\
Input resistance (MOhm) & $710.32 \pm 60.02$ & $707.45 \pm 52.86$ \\
Time constant (ms) & $69.02 \pm 5.36$ & $75.36 \pm 5.88$ \\
AHP (mV) & $23.06 \pm 0.82$ & $25.14 \pm 1.61$ \\
\hline
\end{tabular}

${ }^{* *} p<0.01, * p<0.05$ 